\title{
Computing the starting state for Gibbs-Duhem integration
}

\author{
A. van 't Hof, S. W. de Leeuw, and C. J. Peters ${ }^{\text {a) }}$ \\ Physical Chemistry and Molecular Thermodynamics, Faculty of Applied Sciences, \\ Delft University of Technology, Julianalaan 136, 2628 BL Delft, The Netherlands
}

(Received 4 April 2005; accepted 18 October 2005; published online 3 February 2006)

\begin{abstract}
Gibbs-Duhem integration implies the numerical integration of a Clapeyron equation. To start the numerical integration, an initial coexistence point and a corresponding initial slope of the Clapeyron equation are needed. In order to apply Gibbs-Duhem integration to all kinds of systems at diverse physical conditions, one has to investigate and assess the available methods that can be used to compute these initial values. This publication focuses on vapor-liquid equilibria in binary mixtures comprising chain molecules. The initial coexistence point is either computed with the $N V \beta$ Gibbs ensemble or with the $N p \beta+$ test molecule method with overlapping distributions, which is introduced in this publication. Although computationally demanding, the $N p \beta+$ test molecule method with overlapping distributions is applicable at conditions where the $N V \beta$ Gibbs ensemble fails. We investigated three methods that can be employed to compute the initial slope of the Clapeyron equation. The Widom method and the overlapping-distributions difference method provide correct values for the initial slope. The difference method does only provide the correct answer in special cases. The possibility to judge the reliability of the results makes the overlapping-distributions difference method the safest route to the initial slope. Gibbs-Duhem integration requires the frequent computation of the slope of the Clapeyron equation. This slope depends on ensemble averages of the composition. A new bias method for efficient sampling of the composition in a semigrand-canonical simulation of chain molecules is presented. This bias method considerably enhances the composition sampling in systems comprising chain molecules of different sizes. (C) 2006 American Institute of Physics. [DOI: 10.1063/1.2137705]
\end{abstract}

\section{INTRODUCTION}

Knowledge of physical properties of pure components and mixtures is essential when designing new processes or improving the efficiency of existing processes. Many purecomponent physical properties can be retrieved from literature. However, mixture properties are less often measured and documented. Furthermore, available mixture properties are often measured only at moderate physical conditions. Computer power has increased considerably over the last years. Thus, it is possible to employ computationally demanding methods such as molecular simulations for the prediction of physical properties. This is particularly attractive at physical conditions where real experiments are expensive or simply impracticable.

In this work, we focus on Monte Carlo (MC) molecular simulation of vapor-liquid equilibria in binary mixtures comprising chain molecules. Among the simulation methods that are commonly used to predict vapor-liquid equilibria is the Gibbs-Duhem integration (GDI) method. ${ }^{1,2}$ As opposed to many other simulation techniques, GDI has the advantage of being independent of insertions and/or removals of molecules. This independence makes the method especially suitable for the computation of phase equilibria including a solid phase. ${ }^{3-11}$ In case a good estimate of an initial coexistence

${ }^{a)}$ Electronic mail: c.j.peters@tnw.tudelft.nl point is known, the method is appropriate for the prediction of complete phase diagrams including vapor, liquid, and solid phases. ${ }^{3,7,8}$

A Gibbs-Duhem equation describes the mutual dependence of state variables in an individual thermodynamic phase. Combination of the Gibbs-Duhem equations of coexisting phases enables one to derive a so-called Clapeyron equation. Gibbs-Duhem integration implies the numerical integration of the Clapeyron equation. A Clapeyron equation describes monovariant phase coexistence; it applies to any type of coexistence situation, as long as the number of degrees of freedom is 1 . The original Clapeyron equation describes how the saturation pressure changes with temperature in order for two phases to remain at coexistence. GibbsDuhem integration is easily extended to binary or multicomponent mixtures ${ }^{12}$ and multiphase systems ${ }^{13}$ by deriving the appropriate Clapeyron equation.

The numerical integration traces a coexisting curve from a predetermined initial coexistence point and a corresponding value of the right-hand side of the Clapeyron equation. The right-hand side will be called the initial slope in what follows. In order to correctly implement GDI, good estimates for the initial conditions are needed.

There are several simulation techniques that can be conveniently used to predict the initial coexistence point and the initial slope at moderate conditions, i.e., at moderate molar volumes and for mixtures consisting of small molecules that are not too different. The computation of the initial coexistence point and the corresponding initial slope often requires 
advanced simulation techniques in case the densities of the equilibrium phases differ much, the system contains large chain molecules, and/or the size and force-field parameters of the molecules in the mixture differ much. The prediction of the initial coexistence point and the initial slope with MC molecular simulation for systems containing chain molecules will be discussed in detail in Secs. II A and II B.

The numerical integration of the Clapeyron equation requires the frequent computation of the slope. ${ }^{1,2,12}$ In this work, we focus on vapor-liquid equilibria of binary mixtures. The slope of the corresponding Clapeyron equation is computed with molecular simulation in an isobaric semigrandcanonical ensemble. In this ensemble, the composition fluctuates while the total number of molecules is constant. In Sec. II C, a method is presented that can be used to efficiently sample the composition in a semigrand-canonical ensemble simulation of a system containing chain molecules of different sizes. Section IV contains a summary and concluding remarks.

\section{METHODOLOGY}

\section{A. Computing the initial coexistence point}

The Clapeyron equation that is considered here describes vapor $(V)$-liquid $(L)$ phase coexistence in a binary mixture at constant temperature $\beta=1 /\left(k_{B} T\right), k_{B}$ being Boltzmann's constant $^{12}$

$$
\left(\frac{\partial p}{\partial \xi_{2}}\right)_{\beta, \sigma}=\frac{x_{2}^{L}-x_{2}^{V}}{\xi_{2}\left(1-\xi_{2}\right) \beta\left(v^{L}-v^{V}\right)},
$$

where $\xi_{2}$ is the fugacity fraction of component $2,{ }^{12,14,15} x_{2}$ is the mole fraction of component 2 , and $v$ is the volume per molecule. The subscript $\sigma$ indicates that the derivative is taken along the saturation line. A suitable initial coexistence point for Clapeyron equation (1) is the saturation pressure of one of the pure components, along with the liquid- and vapor-phase densities at coexistence, all evaluated at the temperature of interest.

The appropriateness of a method to compute the initial coexistence point depends on the physical conditions of the systems and on the components of interest. In a previous publication, ${ }^{16}$ we discussed two methods that can be used to compute pure-component coexistence points: the $N V \beta$ Gibbs ensemble method ${ }^{17-22}$ and a modification of the original ${ }^{23,24}$ $N p \beta+$ test molecule method.

The $N V \beta$ Gibbs ensemble method is a very convenient method to compute a vapor-liquid coexistence point, as it provides the liquid- and vapor-phase densities at coexistence along with the saturation pressure at the temperature of interest from a single simulation. Ensemble averages of the vapor- and liquid-phase densities at coexistence are obtained in the usual way by averaging instantaneous densities, which have been sampled at regular intervals. The pressure is computed from the virial for pairwise-additive potentials ${ }^{25}$ or from volume perturbations. ${ }^{26}$ Unfortunately, the applications of the $N V \beta$ Gibbs ensemble are limited to systems with phases of moderate density. At high density, successful exchanges of molecules between the phases are rare; the majority of the insertions of molecules into a high-density phase results in overlap with other molecules. Furthermore, the difference between the liquid- and vapor-phase molar volumes limits the applicability of the $N V \beta$ Gibbs ensemble method, as the total volume and the total number of molecules of both phases are constant.

The basic idea of the original $N p \beta+$ test molecule $\operatorname{method}^{23,24}$ and its extensions ${ }^{16,27-31}$ is the construction of the chemical potential as a function of pressure, temperature, and composition, and the subsequent calculation of the vapor-liquid saturation pressure from the criterion of equality of chemical potentials. Individual $N p \beta$ simulations are performed for a liquid and a vapor phase. As the simulations are performed at constant number of molecules, a large difference between the liquid- and the vapor-phase molar volumes does not limit the application of the method. The liquidphase pressure dependence of the chemical potential is approximated by a Taylor-series expansion truncated after the second term

$$
\begin{aligned}
\beta_{0} \mu^{\prime L}\left(p ; \beta_{0}\right) \approx & \beta_{0} \mu^{\prime L}\left(p_{0}^{L}, \beta_{0}\right)+\beta_{0}\left\langle v^{L}\right\rangle\left(p-p_{0}^{L}\right) \\
& -\frac{N^{L} \beta_{0}^{2}}{2}\left\{\left\langle\left(v^{L}\right)^{2}\right\rangle-\left\langle v^{L}\right\rangle^{2}\right\}\left(p-p_{0}^{L}\right)^{2},
\end{aligned}
$$

where $v^{L}$ is the liquid-phase volume per molecule, $N^{L}$ is the number of molecules in the liquid phase, $\beta_{0}$ is the reciprocal reference temperature, $p_{0}^{L}$ is the liquid-phase reference pressure, and $\langle\cdots\rangle$ is an ensemble average computed at pressure $p_{0}^{L}$ and reciprocal temperature $\beta_{0}$. The prime in Eq. (2) indicates that the purely temperature-dependent part of the idealgas chemical potential has been excluded; i.e., $\mu^{\prime}$ is a configurational chemical potential. According to Vrabec and Fischer, ${ }^{27,30,31}$ it is convenient to treat the ideal-gas chemical potential analytically and to use a truncated Taylor-series expansion for the residual chemical potential. This approximation reveals the logarithmic dependence of the vapor-phase configurational chemical potential on the pressure

$$
\begin{aligned}
\beta_{0} \mu^{\prime V}\left(p ; \beta_{0}\right) \approx & \beta_{0} \mu_{0}^{\prime V}\left(p_{0}^{V} ; \beta_{0}\right)+\left(\beta_{0}\left\langle v^{V}\right\rangle-\frac{1}{p_{0}^{V}}\right)\left(p-p_{0}^{V}\right) \\
& +\ln \left(\frac{p}{p_{0}^{V}}\right)
\end{aligned}
$$

where $v^{V}$ is the vapor-phase volume per molecule, $p_{0}^{V}$ is the reference pressure of the vapor phase, and $\langle\cdots\rangle$ is an ensemble average computed at pressure $p_{0}^{V}$ and reciprocal temperature $\beta_{0}$.

From Eqs. (2) and (3), it is clear that the computation of the coexistence condition from the intersection of the Taylorseries expansions requires knowledge of the configurational chemical potentials $\mu_{0}{ }^{\prime}$ at a reference temperature and pressure. The modification of the original $N p \beta+$ test molecule method that was presented in a previous publication ${ }^{16}$ comprises a simulation scheme that enables the prediction of the pure-component saturation pressure even from a bad initial guess of the true saturation pressure. The chemical potential was computed from the Rosenbluth factor, ${ }^{32}$ which made the method applicable at relatively high densities. In this paper, we further extend the applicability of the modified $N p \beta$ + test molecule method, while using the same iterative 
scheme to arrive at the saturation pressure. The configurational chemical potential is now computed from the overlapping-distributions method of Shing and Gubbins ${ }^{33,34}$ and from the Rosenbluth factor. ${ }^{32,35}$ The combination of the overlapping-distributions method of Shing and Gubbins and Rosenbluth sampling is applicable at even higher densities and provides the possibility to judge the reliability of the answer.

Below, a derivation is given for the distributions that are needed to compute the configurational chemical potentials $\mu_{0}^{\prime}$ at the reference point from the Shing-Gubbins overlapping-distributions method in the $N p \beta$ ensemble. In order to simplify the notation of the derivations, we first discuss how ideal-chain intramolecular configurations are generated. An ideal-chain molecule is an ideal-gas molecule with neither intramolecular Lennard-Jones nor intramolecular Coulombic interactions. The total interaction energy of a system of molecules is separated into three contributions; $\mathcal{U}^{\text {ext }}$ is the sum of the intermolecular Lennard-Jones $(\mathrm{LJ})$ and Coulombic $(C)$ interaction energies, $\mathcal{U}^{\text {intra }}$ describes the contributions from bond-angle bending and rotations about tor-

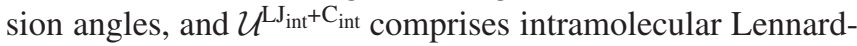
Jones and Coulombic interactions.

Ideal-chain intramolecular configurations can be efficiently generated in accordance with their Boltzmann weight, $\propto \exp \left(-\beta \mathcal{U}^{\text {intra }}\right)$, using a rejection method. ${ }^{32}$ The probability to generate an ideal-chain intramolecular configuration between $\boldsymbol{\Psi}$ and $\boldsymbol{\Psi}+d \boldsymbol{\Psi}$ is given by

$$
d \Psi=\frac{\exp \left(-\beta \mathcal{U}^{\text {intra }}\right) J d \mathbf{q}^{n-3} d \mathbf{s}^{3}}{z},
$$

where $J$ is the Jacobian of transformation from Cartesian to generalized coordinates, $z$ is a normalization constant, $\mathbf{s}^{3}$ represents the vector of overall translational coordinates, scaled by the length of the cubic simulation box, and $\mathbf{q}^{n-3}$ represents the configuration vector of a molecule with $n$ degrees of freedom. Notice that the intramolecular Lennard-Jones and Coulombic interactions are left out of probability distribution (4). The reason is that it is computationally very demanding, especially for large chain molecules, to compute intramolecular Lennard-Jones and Coulombic energies for every generated trial configuration. Although the molecules that are used in this paper are far from large, the intramolecular Lennard-Jones and Coulombic energies are left out of probability distribution (4) to keep the method generally ap- plicable. In case the intramolecular configurations are generated according to probability distribution (4), the $N p \beta$ partition function can be written as follows ${ }^{36}$

$$
\begin{aligned}
& \Delta_{N p \beta}^{*}=\frac{(z q)^{N} N}{N !} \int d V \frac{V^{N}}{V} \exp (-\beta p V)
\end{aligned}
$$

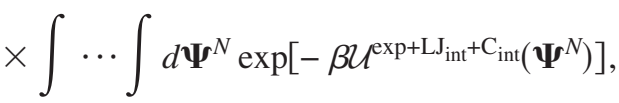

where $q$ is the kinetic contribution of one molecule to the partition function and $N$ is the number of molecules. There is one subtle difference between partition function (5) and its counterparts presented in several text books; ${ }^{25,32}$ Eq. (5) contains a factor $N V^{-1}$ instead of an undetermined volume scale that keeps the partition function dimensionless. ${ }^{37}$ Partition function (5) can be derived from the general $N p \beta$ partition function as presented by $\operatorname{Corti}^{38,39}$ and by Corti and Soto-Campos, ${ }^{40}$ who use a shell molecule to explicitly define the simulation volume. Without this shell molecule, an isobaric partition function redundantly counts volume states. The volume scale in the general $N p \beta$ partition function depends on the interactions of the system with the surroundings as well as on the shape of the simulation box. In case the system is homogeneous, interactions with the surroundings are negligible and cubic periodic boundary conditions are used, the general $N p \beta$ partition function reduces to Eq. (5). ${ }^{37,41}$ The asterisk in Eq. (5) is meant to distinguish the corrected partition function from a conventional $N p \beta$ partition function.

The derivation of the overlapping distributions of the Shing-Gubbins method in the $N p \beta$ ensemble starts by defining a perturbed and an unperturbed system. The unperturbed or reference system (subscript "ref") contains $N$ real molecules and an ideal-chain molecule, while the perturbed system (subscript "per") comprises $N+1$ real molecules. The variable that needs to be sampled in the perturbed and in the unperturbed system is given by

$$
\mathcal{X}=\beta \Delta \mathcal{U}_{\mathrm{test}}^{\mathrm{ext}+\mathrm{LJ} \mathrm{J}_{\mathrm{int}}+\mathrm{C}_{\mathrm{int}}}-\ln \left(\frac{\beta p V}{N+1}\right),
$$

where $\Delta \mathcal{U}_{\text {test }}^{\text {ext }+\mathrm{L}_{\text {int }}+C_{\text {int }}}$ is the energy change that occurs when an ideal-chain molecule is changed into a real molecule. The probability density to find the unperturbed system in "state" $\mathcal{X}$ is derived from Eq. (5)

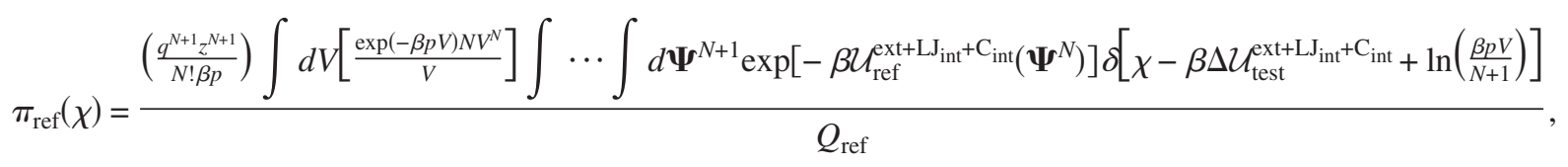

where $Q_{\text {ref }}$ is a normalization constant. The factor $N$ ! instead of $(N+1)$ ! in Eq. (7) is due to the fact that the ideal-chain molecule can be distinguished from the real molecules. The counterpart of Eq. (7) in the perturbed system is given by 


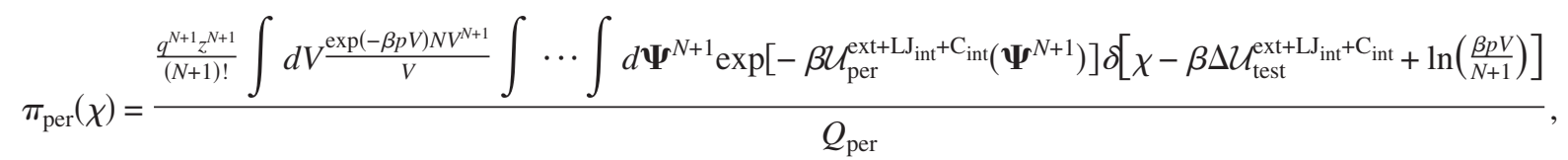

The factor $q^{N+1} z^{N+1}$ is omitted for convenience in what follows. The $\delta$ function in Eq. (8) allows substituting $\chi$

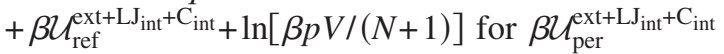

$$
\pi_{\mathrm{per}}(\chi)
$$

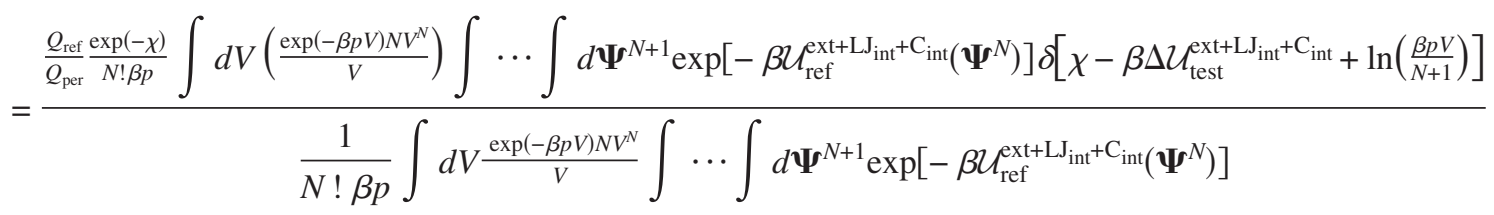$$
=\frac{Q_{\text {ref }}}{Q_{\text {per }}} \exp (-\chi) \pi_{\text {ref }}(\chi) \text {. }
$$

The ratio of normalization constants $Q_{\text {per }} / Q_{\text {ref }}$ can be related to the configurational chemical potential $\mu^{\prime}$

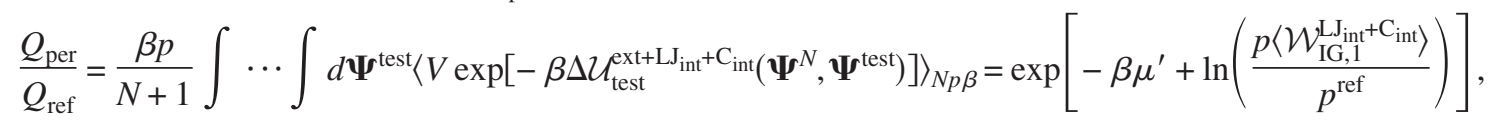

where $\left\langle\mathcal{W}_{\mathrm{IG}, i}^{\mathrm{LJ}, i} \mathrm{C}_{\text {int }}\right\rangle$ is the normalized Rosenbluth factor of an isolated chain of type $i .^{32,42}$ This normalized Rosenbluth factor is given by

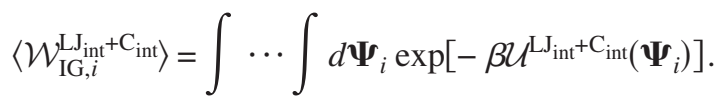

Insertion of Eq. (10) into Eq. (9) gives

$$
\ln \pi_{\mathrm{per}}(\chi)=\beta \mu^{\prime}-\ln \left(\frac{p\left\langle\mathcal{W}_{\mathrm{IG}, 1}^{\left.\mathrm{LJ}_{\mathrm{in}}+\mathrm{C}_{\mathrm{int}}\right\rangle}\right.}{p^{\text {ref }}}\right)-\chi+\ln \pi_{\mathrm{ref}}(\chi) .
$$

Notice that the Rosenbluth factor of an isolated chain, which is a purely temperature-dependent property, is not needed in the computation of phase equilibria, as it is equal in coexisting phases. The actual value of the configurational chemical potential is obtained from a graphical analysis of the difference between the following distributions

$$
f_{\text {per }}=\ln \pi_{\text {per }}(\chi)+\frac{1}{2} \chi, \quad f_{\text {ref }}=\ln \pi_{\text {ref }}(\chi)-\frac{1}{2} \chi .
$$

Equation (12) is similar to the relation presented by Sindzingre et $a l^{43}$ who derived it for single-site Lennard-Jones molecules. Kofke and Glandt also used a similar relation in order to compute pure-component fugacities of LennardJones systems. ${ }^{14}$ They used a joint energy-volume distribution, which provides the same result but requires longer simulations.

The overlapping-distributions method requires simple test-molecule insertions and test removals of real molecules in two separate simulations. In this paper, Rosenbluth sampling is used to compute the configurational chemical potential. The combination of Rosenbluth sampling and the overlapping-distributions method has been worked out by
Mooij and Frenkel. ${ }^{35}$ The working equation that is used to obtain the configurational chemical potential is identical to Eq. (12), however, the variable that must be sampled is now

$$
\chi=-\ln \mathcal{W}^{\text {ext+LJ } \mathrm{Lint}_{\text {int }}+\mathrm{C}_{\mathrm{int}}}-\ln \left(\frac{\beta p V}{N+1}\right),
$$

where $\mathcal{W}^{\text {ext+LJ }}$ int $+C_{\text {int }}$ is the Rosenbluth factor of a test molecule with intermolecular interactions. ${ }^{32,35}$ The possibility to compute the configurational chemical potential from the overlapping-distributions method depends on two factors. At first, as the name of the method indicates, the distributions $f_{\text {per }}$ and $f_{\text {ref }}$ should have a region of overlap. Secondly, the difference between the distributions should give a constant value of $\beta \mu^{\prime}$ over a range of $\chi$ values.

\section{B. Initial slope of the Clapeyron equation}

As pointed out in Sec. II A, the initial point from which the numerical integration is started is one of the purecomponent vapor-liquid coexistence points at the temperature of interest. However, Clapeyron equation (1) is not defined for a pure component. Fortunately, it is possible to derive an appropriate form of the Clapeyron equation in the limit of infinite dilution of a solute (subscript 2) into a solvent (subscript 1$)^{12}$

$$
\lim _{\xi_{2} \rightarrow 0}\left(\frac{\partial p}{\partial \xi_{2}}\right)_{\beta, \sigma}=\frac{1}{\beta\left(v^{L}-v^{V}\right)}\left[\left(\frac{f_{1}}{H_{2}}\right)^{L}-\left(\frac{f_{1}}{H_{2}}\right)^{V}\right],
$$

where $f_{1}$ is the fugacity of the pure solvent and $H_{2}$ is Henry's constant of the infinitely diluted solute. The ratios $f_{1} / H_{2}$ in 
Eq. (15) can be expressed into the residual chemical potentials of the solvent and the infinitely diluted solute

$$
\left(\frac{f_{1}}{H_{2}}\right)=\lim _{x_{2} \rightarrow 0} \exp \left[-\beta\left(\mu_{2}^{\text {res }}-\mu_{1}^{\text {res }}\right)\right] \text {. }
$$

A residual chemical potential equals the full chemical potential minus its ideal-gas contribution at the same pressure, temperature, and composition. The ratios $f_{1} / H_{2}$ can be computed from an ensemble average by perturbing the composition during a simulation in an $N_{1} p \beta$ ensemble. The situation of infinite dilution is approximated by the smallest possible mole fraction in a simulation, i.e., $x_{2}=1 / N$. In what follows, we will discuss three potential methods for the computation of the residual chemical-potential difference $\Delta \mu_{21}^{\text {res }}=\mu_{2}^{\text {res }}$ $-\mu_{1}^{\text {res }}$.

\section{Widom test-molecule insertions}

The most understandable method for the computation of the residual chemical-potential difference $\Delta \mu_{21}^{\text {res }}$ is to separately determine $\mu_{2}^{\text {res }}$ and $\mu_{1}^{\text {res }}$ by Widom test-molecule insertions $^{44,45}$ in the $N_{1} p \beta$ ensemble. In Widom's testmolecule method, molecules that do not influence the evolution of the system are inserted into the simulation box. The individual chemical potentials are computed by ensemble averaging the Boltzmann factors of the interaction energies of the inserted solvent and solute molecules.
In order to facilitate the computation, the residual chemical-potential difference is divided into two contributions. ${ }^{42}$ The first contribution comprises the full chemical-potential difference minus its ideal-chain-gas value (superscript "ic"), while the second contribution consists of the ideal-gas chemical-potential difference (superscript "ig") minus its ideal-chain-gas value

$$
\Delta \mu_{21}^{\text {res, Widom }}=\left(\Delta \mu_{21}-\Delta \mu_{21}^{\text {ic }}\right)^{\text {Widom }}-\left(\Delta \mu_{21}^{\text {ig }}-\Delta \mu_{21}^{\text {ic }}\right) .
$$

The partition functions of an ideal-gas molecule, $Z_{i}^{\mathrm{ig}}$, and of an ideal-chain gas molecule, $Z_{i}^{\text {ic }}$, at constant temperature and pressure are given by

$$
\begin{aligned}
& Z_{i}^{\mathrm{ic}}=\frac{q_{i} z_{i}}{\beta p} \int \cdots \int d \boldsymbol{\Psi}_{i}=\frac{q_{i} z_{i}}{\beta p}, \\
& Z_{i}^{\mathrm{ig}}=\frac{q_{i} z_{i}}{\beta p} \int \cdots \int d \boldsymbol{\Psi}_{i} \exp \left(-\beta \mathcal{U}_{i}^{\mathrm{LJ}} \mathrm{Jin}+\mathrm{C}_{\mathrm{int}}\right) .
\end{aligned}
$$

Having found the ideal-gas and ideal-chain-gas partition functions, we are now able to give a relation for the second contribution on the right-hand side in Eq. (17)

$$
\beta\left(\Delta \mu_{21}^{\mathrm{ig}}-\Delta \mu_{21}^{\mathrm{ic}}\right)=-\ln \left(\frac{Z_{1}^{\mathrm{ic}} Z_{2}^{\mathrm{ig}}}{Z_{2}^{\mathrm{ic}} Z_{1}^{\mathrm{ig}}}\right)=\ln \left(\frac{\left\langle\mathcal{W}_{\mathrm{IG}, 1}^{\mathrm{LJ} \mathrm{J}_{\text {int }}+\mathrm{C}_{\mathrm{int}}}\right\rangle}{\left\langle\mathcal{W}_{\mathrm{IG}, 2}^{\mathrm{LJ}} \mathrm{I}_{\text {int }}+\mathrm{C}_{\mathrm{int}}\right.}\right) .
$$

The first contribution to the residual chemical-potential difference in Eq. (17), in the limit $x_{2} \rightarrow 0$, is given by ${ }^{36}$

$$
\begin{aligned}
& \lim _{x_{2} \rightarrow 0} \beta\left(\Delta \mu_{21}-\Delta \mu_{21}^{\text {ic }}\right)^{\text {Widom }}=-\ln \left[\frac{\beta p}{N_{1}} \int \cdots \int d \boldsymbol{\Psi}_{2}^{\text {test }}\left\langle V \exp \left(-\beta \mathcal{U}_{\text {test }, 2}^{\text {ext }+\mathrm{LJ}_{\text {int }}+\mathrm{C}_{\text {int }}}\right)\right\rangle_{N_{1} p \beta}\right] \\
& +\ln \left[\frac { \beta p } { N _ { 1 } + 1 } \int \cdots \int d \boldsymbol { \Psi } _ { 1 } ^ { \text { test } } \left\langleV \exp \left(-\beta \mathcal{U}_{\text {test }, 1}^{\text {ext } \left.\left.+\mathrm{LJ}_{\text {int }}+\mathrm{C}_{\text {int }}\right)\right\rangle_{N_{1} p \beta}}\right]\right.\right.
\end{aligned}
$$

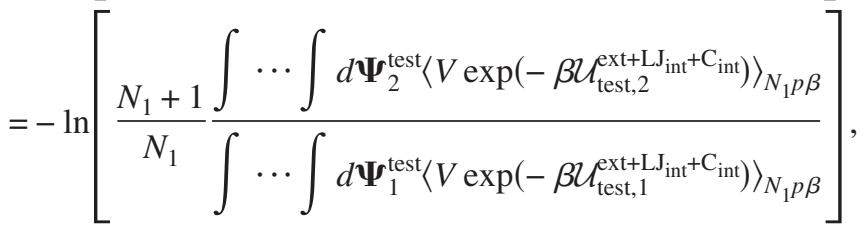

where $\mathcal{U}_{\text {test }, i}^{\text {ext } i \mathrm{LJ}_{\mathrm{int}}+\mathrm{C}_{\text {int }}}$ is the sum of the intermolecular and intramolecular Lennard-Jones and Coulombic energies of a test molecule of identity $i$ and $\boldsymbol{\Psi}_{i}^{\text {test }}$ is a random ideal-chain test-molecule configuration generated in accordance with Eq. (4). The ensemble averages in Eq. (21) are obtained from a single $N_{1} p \beta$ ensemble simulation. The ratio $f_{1} / H_{2}$, computed from Widom's test-molecule method, can now be related to ensemble averages as follows

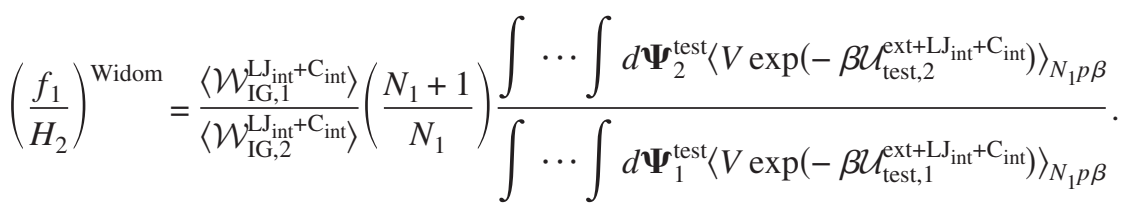

\section{Difference method}

A straightforward perturbation method that can be employed to compute the residual chemical-potential difference is the difference or swap method. The difference method was introduced by Shing ${ }^{46,47}$ in order to compute activity coeffi- cients and residual chemical-potential differences at infinite dilution. The method can be extended to nondiluted mixtures and is also suitable for the computation of other partial molar properties. ${ }^{43,48-55}$ The chemical-potential difference at constant temperature and constant pressure is given by 


$$
\beta \Delta \mu_{21}=-\ln \left(\frac{\Delta_{N_{1}-1, N_{2}+1, p \beta}^{*}}{\Delta_{N_{1} N_{2} p \beta}^{*}}\right),
$$

where $\Delta_{N_{1} N_{2} p \beta}^{*}$ is the partition function of a binary isothermal-isobaric ensemble that correctly counts volume states. The derivations of the residual chemical-potential difference as published by Shing ${ }^{46,47}$ and by Sindzingre et ll $^{43,55}$ are applicable to systems with single-site or rigid molecules. In this publication, a straightforward derivation is given for chain molecules with intramolecular degrees of freedom and intramolecular Lennard-Jones and Coulombic interactions. Employment of Eq. (17) gives the following result for the residual chemical-potential difference at infinite dilution. ${ }^{36}$

$$
\begin{aligned}
& \lim _{x_{2} \rightarrow 0} \exp \left(-\beta \Delta \mu_{21}^{\text {res,Diff }}\right) \\
& =\frac{\left\langle\mathcal{W}_{\mathrm{IG}, 1}^{\left.\mathrm{L} \mathrm{J}_{\text {in }}+\mathrm{C}_{\text {int }}\right\rangle}\right.}{\left\langle\mathcal{W}_{\mathrm{IG}, 2}^{\left.\mathrm{LJ} \mathrm{J}_{\text {int }}+\mathrm{C}_{\text {int }}\right\rangle}\right.} \int \cdots \int d \boldsymbol{\Psi}_{2}^{\text {test }}
\end{aligned}
$$

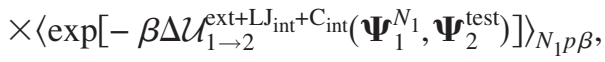

where $\boldsymbol{\Psi}_{i}^{\text {test }}$ is a random ideal-chain test-molecule configuration, generated in accordance with Eq. (4), and $\Delta \mathcal{U}_{1 \rightarrow 2}^{\text {ext+L } \mathrm{J}_{\text {int }}+\mathrm{C}_{\text {int }}}$ is the change in interaction energy that results from converting a molecule of type 1 into a molecule of type 2 .

The simulation procedure is as follows. A single purecomponent $N_{1} p \beta$ ensemble simulation is performed. At regular intervals, one of the molecules is converted into a molecule of type 2 and the exchange energy $\Delta \mathcal{U}_{1 \rightarrow 2}^{\text {ext }+\mathrm{LJ}_{\mathrm{int}}+\mathrm{C}_{\text {int }} \text { is }}$ noted. The new molecule with identity 2 and the old molecule with identity 1 have the same center-of-mass coordinates. The molecule is converted back before the simulation proceeds. The ratio $f_{1} / H_{2}$, computed from the difference method, can now be related to an ensemble average of a Boltzmann factor as follows

$$
\begin{aligned}
& \left(\frac{f_{1}}{H_{2}}\right)^{\text {Diff }}=\frac{\left\langle\mathcal{W}_{\mathrm{IG}, 1}^{\left.\mathrm{LJ}_{\mathrm{in}}+\mathrm{C}_{\text {int }}\right\rangle}\right.}{\left\langle\mathcal{W}_{\mathrm{IG}, 2}^{\left.\mathrm{LJ}_{\mathrm{int}}+\mathrm{C}_{\text {int }}\right\rangle}\right.} \int \cdots \int d \boldsymbol{\Psi}_{2}^{\text {test }}
\end{aligned}
$$

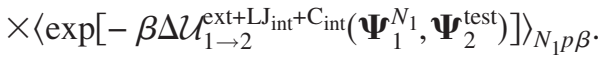

\section{Overlapping-distributions difference method}

Shing and Gubbins ${ }^{33,34}$ and Powles et al. ${ }^{56}$ showed how to combine test-molecule insertions and test removals of real molecules in order to compute the chemical potential from a Monte Carlo simulation. The Shing-Gubbins method, which is also called $f$-g method, is a special case of the curve-fitting method, which has been developed by Bennett ${ }^{57}$ for computing the free-energy difference between two systems.

Sindzingre et al. ${ }^{43,55}$ suggested the combination of the difference method and the $f$ - $g$ method for obtaining accurate simulation results for residual chemical-potential differences and other partial molar properties. $\mathrm{Liu}^{48}$ and Liu and Govind $^{49,50}$ actually performed computations with this com- bination of methods. The combination of methods will be called overlapping-distributions difference method in this publication.

Below, a derivation is given for the overlapping distributions of the difference method. The unperturbed (reference) system has $N_{1}$ molecules of type $1, N_{2}$ molecules of type 2 , and an ideal-chain molecule of type 2 . The perturbed system comprises $N_{1}-1$ molecules of type $1, N_{2}+1$ molecules of type 2 , and an ideal-chain molecule of type 1 . The variable that needs to be sampled in both the reference and the perturbed systems is ${ }^{36}$

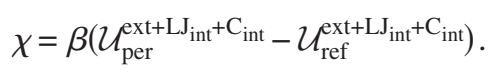

The procedure to derive the residual chemical-potential difference is similar to the one given in Sec. II A. The relation between the overlapping distributions $\pi_{\text {per }}$ and $\pi_{\text {ref }}$ is given by $^{36}$

$$
\begin{aligned}
& \ln \pi_{\mathrm{per}}(\chi)+\frac{1}{2}\left(\ln \frac{\left\langle\mathcal{W}_{\mathrm{IG}, 2}^{\left.\mathrm{LJ} \mathrm{Ju}_{\mathrm{in}}+\mathrm{C}_{\mathrm{int}}\right\rangle}\right.}{\left\langle\mathcal{W}_{\mathrm{IG}, 1}^{\mathrm{LI}, \mathrm{in}}+\mathrm{C}_{\mathrm{int}}\right\rangle}+\chi\right)=\beta \Delta \mu_{21}^{\mathrm{res}, \mathrm{OlD}}
\end{aligned}
$$

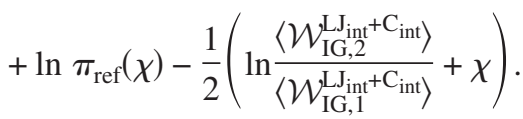

The residual chemical-potential difference is obtained from a graphical analysis of the following distributions

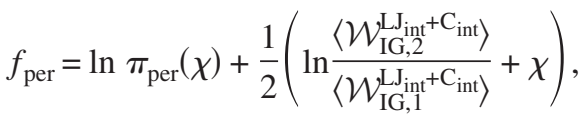

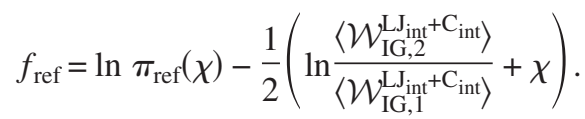

The ratio $\left(f_{1} / H_{2}\right)^{\mathrm{OID}}$, computed from the overlappingdistributions difference method, is obtained from Eq. (16) and from $\beta \Delta \mu_{21}^{\text {res, OlD }}$ in the limit of infinite dilution.

\section{Energy-biased identity changes in the semigrand- canonical ensemble}

Numerical integration of Clapeyron equation (1) requires the computation of its slope at several state points. The variables that occur in the slope of Clapeyron equation (1) correspond to an isobaric semigrand-canonical ensemble. ${ }^{14}$ The values of the liquid- and vapor-phase volumes and mole fractions at constant number of molecules, temperature, pressure, and fugacity fraction are computed from binary isobaric semigrand-canonical simulations.

An advantage of a semigrand-canonical-ensemble simulation is the independence of insertions and removals of molecules. The method requires identity changes of molecules 
instead. Such an identity change consists of the deletion of a molecule with identity $i$ and the generation of a new molecule with identity $j$ at the same center-of-mass coordinates. The identity changes may be very efficient in case molecules of type $i$ and type $j$ have similar size, shape, and force-field parameters. However, for real molecules these differences are generally not negligible. They often have a dramatic effect on the fraction of accepted identity changes. It shall be clear that a simple implementation of the identity change that is performed between two very different molecules seldom results in an accepted new state. Employing a bias method may increase the number of accepted identity changes and the "diffusion through composition space."

A bias method uses information on the present configuration of molecules for the selection of a favorable new configuration. Before deriving the acceptance criterion for a biased identity change, we discuss the isobaric semigrandcanonical partition function. According to Frenkel and Smit, $^{32}$ the isobaric semigrand-canonical partition function $\mathcal{Y}_{N p \beta \xi_{2}}^{\prime}$ for a binary mixture can be written as follows

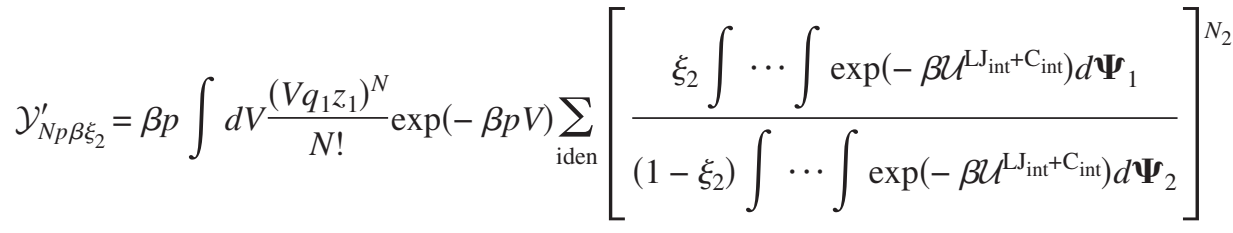

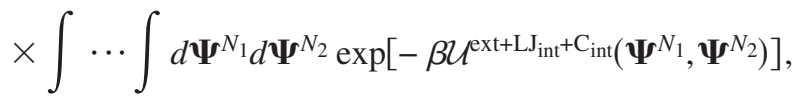

where $z_{1}$ is a normalization constant of the intramolecular probability distribution of molecules of type 1 [see Eq. (4)] and $q_{1}$ is the kinetic contribution of a molecule of type 1 to the partition function. The summation indicated by "iden" represents a sum over all possible identities of all molecules. ${ }^{14,32}$ The free energy belonging to partition function (29) is the effective full semigrand energy $\beta N \mu_{1}$. As pointed out in Sec. II A, conventional isobaric partition functions, like the semigrand-canonical one in Eq. (29), redundantly count volume states. ${ }^{38-40}$ In this paper, a shifted partition function $\mathcal{Y}_{N p \beta \xi_{2}}^{*}$ is used that correctly counts volume states and relates to the effective configurational semigrand energy $N \ln \left(\hat{f}_{1} / p^{\text {ref }}\right)($ Ref. 36)

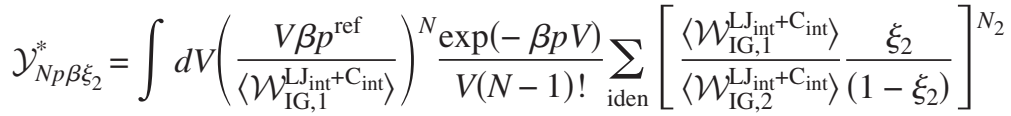

$$
\begin{aligned}
& \times \int \cdots \int d \boldsymbol{\Psi}^{N_{1}} d \boldsymbol{\Psi}^{N_{2}} \exp \left[-\beta \mathcal{U}^{\text {ext+LJ }} \mathrm{Lint}+\mathrm{C}_{\mathrm{int}}\left(\boldsymbol{\Psi}^{N_{1}}, \boldsymbol{\Psi}^{N_{2}}\right)\right],
\end{aligned}
$$

where $p^{\text {ref }}$ is a reference pressure, the value of which is unimportant. The asterisk in Eq. (30) is again meant to distinguish the corrected partition function from a conventional isobaric semigrand-canonical partition function. Imposing the criterion of detailed balance ${ }^{25,32}$ to an identity-change MC move in the binary isobaric semigrand-canonical ensemble gives the following acceptance probability. ${ }^{36}$

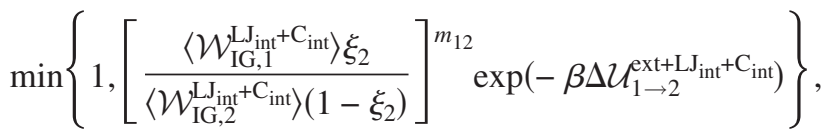

where $m_{12}=-1$ for identity changes $2 \rightarrow 1$ and $m_{12}=+1$ for

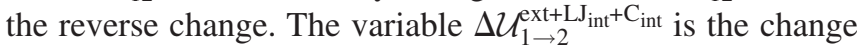
in interaction energy that results from the identity change.

As already mentioned above, simply using acceptance criterion (31) will result in only a small fraction of accepted identity changes in case molecules of types 1 and 2 are very different. In an unbiased sampling scheme, ${ }^{14}$ a molecule is picked randomly and an identity change is performed. In this paper, the choice of the molecule whose identity is to be changed is chosen based on the energies of the molecules that constitute the system. This choice of bias is based on the following considerations. The energy of a settled molecule

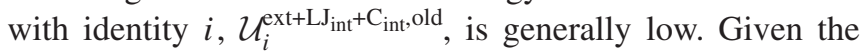
energy of the settled molecule, the acceptance probability (31) for the identity change is highest for new molecules

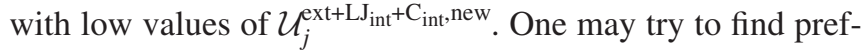
erential orientations for a molecule of type $j$ in an orientational-bias fashion. However, it is much easier to choose settled configurations with a high energy $\mathcal{U}_{i}^{\text {ext }+\mathrm{LJ}_{\text {int }}+\mathrm{C}_{\text {int }} \text {, old }}$; although $\mathcal{U}_{i}^{\text {ext }+\mathrm{LJ}_{\text {int }}+\mathrm{C}_{\text {int }} \text {,old }}$ is generally low, the higher its value, the larger the acceptance probability of the identity-change MC move.

In case the number of molecules that takes identity $j$ is small compared to the total number of molecules, for example, at $\xi_{j} \ll 0.5$, it may be worthwhile to perform the identity change $j \rightarrow i$ more often than is justified based on the mole fraction of component $j$. This can be accomplished by introducing the fugacity fraction into the selection criterion of a molecule. The above-mentioned criteria result in the following biased probability to perform an identity-change MC move on molecule $i$ 


$$
\begin{aligned}
\pi_{i}= & \frac{\exp \left[+\gamma \beta \mathcal{U}_{i}^{\text {ext }+\mathrm{L}_{\text {int }}+\mathrm{C}_{\text {int }} \text { old }}-\omega m_{i} \ln \left(\xi_{2} /\left(1-\xi_{2}\right)\right)\right]}{\sum_{j=1}^{N} \exp \left[+\gamma \beta \mathcal{U}_{j}^{\text {ext }+\mathrm{LJ}_{\text {int }}+\mathrm{C}_{\text {int }} \text { old }}-\omega m_{j} \ln \left(\xi_{2} / 1-\xi_{2}\right)\right]} \\
= & \frac{\mathrm{C}_{i}^{\text {old }}}{\sum_{j=1}^{N} \mathrm{C}_{j}^{\text {old }}},
\end{aligned}
$$

where $m_{i}=-1$ in case molecule $i$ is of identity 1 and $m_{i}$ $=+1$ when molecule $i$ is of identity 2 . The parameters $\gamma$ and $\omega$ determine the strength of the bias. The exponents in Eq. (32) have bound values since the energies of settled molecules are finite negative up to slightly positive. To obey the criterion of detailed balance, ${ }^{25,32}$ the acceptance criterion (31) has to be changed as well. The acceptance criterion for the energy-biased identity change is given by

$$
\begin{aligned}
& \min \left\{1,\left(\frac{\left\langle\mathcal{W}_{\mathrm{IG}, 1}^{\mathrm{LJ}_{\mathrm{in}}+\mathrm{C}_{\text {int }}}\right\rangle}{\left\langle\mathcal{W}_{\mathrm{IG}, 2}^{\mathrm{LJ} \mathrm{J}_{\mathrm{int}}+\mathrm{C}_{\mathrm{int}}}\right.}\right)^{m_{12}} \frac{\sum_{j=1}^{N} \mathrm{C}_{j}^{\text {old }}}{\sum_{j=1}^{N} \mathrm{C}_{j}^{\text {new }}} \times \exp [-(1-\gamma)\right. \\
& \left.\left.\times \beta \Delta \mathcal{U}^{\text {ext }+\mathrm{LJ}_{\mathrm{int}}+\mathrm{C}_{\mathrm{int}}}+(1+2 \omega) m_{12} \ln \left(\frac{\xi_{2}}{1-\xi_{2}}\right)\right]\right\} .
\end{aligned}
$$

\section{RESULTS AND DISCUSSION}

\section{A. Simulation details}

The force field that is used in this work is given by a Lennard-Jones 12-6 potential with Coulombic charges on the interaction sites wherever necessary. The Coulombic interactions were computed with the Ewald summation method. ${ }^{25}$ The Ewald screening parameter and the upper bound in the reciprocal space were fixed at $\alpha=5.6$ and $k_{\max }=6$. The total intermolecular potential is given by

$$
\begin{aligned}
\mathcal{U}^{\text {ext }}= & 4 \sum_{i=1}^{N-1} \sum_{j=i+1}^{N} \sum_{\gamma=1}^{n_{i}} \sum_{\delta=1}^{n_{j}} \varepsilon_{\gamma \delta}\left[\left(\frac{\sigma_{\gamma \delta}}{r_{i \gamma j \delta}}\right)^{12}-\left(\frac{\sigma_{\gamma \delta}}{r_{i \gamma j \delta}}\right)^{6}\right] \\
& +\frac{1}{4 \pi \varepsilon_{0}} \sum_{i=1}^{N-1} \sum_{j=i+1}^{N} \sum_{\gamma=1}^{n_{i}} \sum_{\delta=1}^{n_{j}} \frac{q_{i}^{\gamma} q_{j}^{\delta}}{r_{i \gamma j \delta}}
\end{aligned}
$$

where $\varepsilon_{0}$ is the vacuum permittivity, $q_{i}^{\gamma}$ is partial charge $\gamma$ on molecule $i, n_{i}$ is the number of partial charges on molecule $i, r_{i \gamma j \delta}$ is the distance between interaction sites $i \gamma$ and $j \delta$, and $\sigma_{\gamma \delta}$ and $\varepsilon_{\alpha \gamma}$, respectively, are the Lennard-Jones collisiondiameter and well-depth parameters for the interaction between site $\gamma$ and site $\delta$. The Lennard-Jones parameters for interactions between unlike interaction sites were computed with the Lorentz-Berthelot combining rules. ${ }^{25}$

In this paper, we present simulation results for several binary mixtures. Force-field parameters for the molecules of interest were taken from literature. Methane, ethane, and propane were modeled with the TraPPE-EH force field (explicit- hydrogen transferrable potentials for phase equilibria), ${ }^{58}$ carbon dioxide with the TraPPE-AA (all-atom transferable potential for phase equilibria) force field, ${ }^{59}$ dimethyl sulfoxide (DMSO) with the potential of Rao and Singh, ${ }^{60}$ difluoromethane with the force field of Higashi and Takada, ${ }^{61}$ and trifluoromethane with the force field of Song et al. ${ }^{62}$ Intramolecular flexibility, if present, comprised bond-angle bending and rotation about torsion angles

$$
\begin{aligned}
\mathcal{U}^{\text {intra }}= & \frac{k_{\theta}}{2}\left(\theta-\theta_{0}\right)^{2}+c_{1}[1+\cos \phi]+c_{2}[1-\cos 2 \phi] \\
& +c_{3}[1+\cos 3 \phi],
\end{aligned}
$$

where $\theta$ is a bond angle, $\theta_{0}$ is the expectation value of bond angle $\theta$, and $\phi$ is a torsion angle. Among the abovementioned force fields, only the TraPPE-EH force field has intramolecular degrees of freedom. ${ }^{58}$ The types of molecules that are used in this work have neither intramolecular Lennard-Jones nor intramolecular Coulombic interactions.

In all $N p \beta$ simulations, in the semigrand-canonical simulations, and in the $N V \beta$ Gibbs ensemble simulations in which the pressure was computed from the virial, molecular translations and rotations were, respectively, force biased and torque biased. $^{16,63,64}$ The bias strengths $\lambda$ and $\xi$ were fixed at 0.5 . Cubic periodic-boundary conditions with the minimumimage convention were employed. ${ }^{25}$ The standard LennardJones long-range corrections were applied ${ }^{25}$ with a potential cutoff of three times the largest Lennard-Jones collision diameter $\sigma$ in the simulated system. The cutoff was checked against half the box length after every successful volume change.

Every simulation consisted of 5000 equilibration $\mathrm{MC}$ cycles and 100000 production MC cycles. Maximum translations, rotations, and volume changes were adjusted during the equilibration period of the simulation to yield a fraction of accepted MC trial moves of about 50\%. Different maximum displacements were used for the liquid and the vapor phase. Initially, the statistical errors of the ensemble averages were computed with the block-averaging method of Flyvbjerg and Petersen. ${ }^{65}$ This analysis revealed the minimum block length needed to obtain uncorrelated blocks of samples. It turned out that blocks of a few thousands MC cycles were uncorrelated. The statistical errors given in this work have been computed from application of the bootstrap method $^{66}$ to regularly saved blocks of $2500 \mathrm{MC}$ cycles.

The pure-component coexistence conditions were either computed with a $N V \beta$ Gibbs ensemble simulation or from the $N p \beta+$ test molecule method with overlapping distributions. The performance of the energy-bias method was tested in semigrand-canonical simulations. The MC cycles in these simulation techniques comprise different MC moves.

$N V \beta$ Gibbs ensemble simulations were performed with $N=400$ molecules. The initial densities of the liquid and the vapor phase were chosen such that the vapor phase contained on average approximately 50 molecules. We could accomplish this by changing the initial number of molecules in the liquid $N^{L}$ and in the vapor phase $N^{V}=N-N^{L}$ and/or by changing the initial densities of the liquid and the vapor phase. Samples were taken during the production phase. Ev- 
TABLE I. Simulation results for pure-component saturation pressures and/or effective configurational chemical potentials $\beta \mu^{\prime}$ along with statistical errors at vapor-liquid coexistence conditions.

\begin{tabular}{|c|c|c|c|c|c|c|}
\hline \multirow[b]{2}{*}{ System/condition } & \multicolumn{2}{|c|}{ Gibbs ensemble/virial } & \multicolumn{2}{|c|}{ Gibbs ensemble/volume perturbations } & \multicolumn{2}{|c|}{$N p \beta+\mathrm{TM} /$ overlapp. distributions } \\
\hline & $\begin{array}{c}p^{L, \text { sat }} \\
(\mathrm{MPa})\end{array}$ & $\begin{array}{c}p^{V, \text { sat }} \\
(\mathrm{MPa})\end{array}$ & $\begin{array}{c}p^{L, \text { sat }} \\
(\mathrm{MPa})\end{array}$ & $\begin{array}{c}p^{V, \text { sat }} \\
(\mathrm{MPa})\end{array}$ & $\begin{array}{c}p^{\mathrm{sat}} \\
(\mathrm{MPa})\end{array}$ & $\begin{array}{c}\beta \mu^{\prime} \\
(-)\end{array}$ \\
\hline $\mathrm{C}_{2} \mathrm{H}_{6} / 250 \mathrm{~K}$ & $1.38(7)$ & $1.323(5)$ & $1.37(5)$ & $1.312(7)$ & & \\
\hline $\mathrm{CO}_{2} / 280 \mathrm{~K}$ & $3.84(9)$ & $4.09(5)$ & $4.08(6)$ & $4.03(2)$ & & \\
\hline $\mathrm{C}_{2} \mathrm{H}_{6} / 144.26 \mathrm{~K}$ & & & & & $0.0076(7)$ & $-8.9(9)$ \\
\hline $\mathrm{DMSO} / 278.5 \mathrm{~K}$ & & & & & $0.0017(1)$ & $-10.8(9)$ \\
\hline DMSO/303.15 K & & & & & $0.0063(5)$ & $-9.57(8)$ \\
\hline $\mathrm{DMSO} / 328.94 \mathrm{~K}$ & & & & & $0.019(1)$ & $-8.55(7)$ \\
\hline
\end{tabular}

ery MC cycle in a $N V \beta$ Gibbs ensemble simulation consisted of $0.7 \mathrm{~N}$ translations, $0.3 \mathrm{~N}$ rotations, a number of exchanges of molecules between the phases, and 2 or 6 changes of the coupled volumes.

The saturation pressure was either computed from volume perturbations or from the virial. The number of volume changes per $\mathrm{MC}$ cycle was 2 for pressure computation from the virial and 6 for pressure computation from volume perturbations.

The configurational-bias method was used to achieve an acceptable percentage of successful exchanges of molecules between the coexisting phases. ${ }^{32}$ The number of attempted exchanges of molecules between the phases and the number of trials in the growth process ${ }^{32}$ of a molecule were adjusted such that the fraction of accepted exchanges was approximately between $2 \%$ and $5 \%$.

In all $N p \beta$ simulations and in the semigrand-canonical simulations, liquid phases contained $N^{L}=300$ molecules and vapor phases $N^{V}=100$ molecules. Every MC cycle consisted of $0.7 N^{i}$ translations, $0.3 N^{i}$ rotations, and 1 volume change, where $i$ represents the liquid or the vapor phase. In the semigrand-canonical simulations, we also performed $0.5 N^{i}$ energy-biased identity changes.

The $N p \beta$ simulations that were performed to compute the initial slope of the Clapeyron equation required several extra perturbations. Application of the Widom method required $0.5 N^{i}$ test insertions for molecules of both components. In the application of the difference method, we included $N^{i}$ test-identity changes, and in the overlappingdistributions difference method we included $0.5 N^{i}$ test removals of real molecules or $0.5 N^{i}$ test insertions of molecules. The distributions of the overlapping-distributions difference method contained 201 bins. The bin width was determined during the equilibration phase of the simulation.

\section{B. Simulation results}

\section{Initial point}

The suitability of a simulation method to compute the initial coexistence point depends on a number of factors. The most important one is the capability of the method to predict an accurate answer. Other factors are the required simulation length for obtaining a reliable result and the complexity of the computer code. Simulation results for several purecomponent vapor-liquid coexistence points are given in Table I. Only results for saturation pressures are presented, as they completely define the condition of phase coexistence at the temperature of interest. Densities at vapor-liquid coexistence can be computed from subsequent $N p \beta$ simulations. We will not compare the performance of the $N p \beta+$ test molecule method with overlapping distributions and $N V \beta$ Gibbs ensemble simulations, as these methods are completely different. The applicability of the methods at different physical conditions will be discussed instead.

The saturation pressures in $N V \beta$ Gibbs ensemble simulations were computed in both liquid and vapor phases, from either the virial ${ }^{25}$ or volume perturbations. ${ }^{26}$ As pointed out in Sec. II A, the $N V \beta$ Gibbs ensemble can be conveniently used when the liquid-phase density and the difference between the liquid- and the vapor-phase molar volumes are moderate. In case the liquid-phase density is moderate, it is worthwhile to use force-biased translations and torquebiased rotations in order to enhance the diffusion through configuration space. ${ }^{16,63,64}$ These bias methods require the computation of intermolecular forces. If the force between the molecules is computed, it requires little effort to compute the virial as well. Thus, at moderate densities, computing the pressure from the virial is convenient.

On the other hand, in case the liquid phase is far from dense, force and torque-biased methods are less relevant and computing the pressure from volume fluctuations is an attractive alternative, since $N V \beta$ Gibbs ensemble simulations already require volume trial moves. However, as the computation of the pressure from volume fluctuations requires small volume perturbations, ${ }^{26}$ an increased number of volume trial moves is necessary to sample all accessible volume states within a finite number of MC cycles. An advantage of computing the saturation pressure from volume perturbations is that it may provide reliable results from a liquid-phase simulation as well as from a vapor-phase simulation. This is often not possible within reasonable time in case the saturation pressure is computed from the virial. Getting rid of the virial is particularly advantageous when the Ewaldsummation method ${ }^{25}$ is used to compute the Coulombic interactions as the evaluation of the Coulombic forces between molecules is computationally very demanding.

The moving ensemble averages of the liquid- and vaporphase saturation pressures, computed with both methods, are given in Fig. 1. The results were obtained for pure ethane ${ }^{58}$ at $T=250 \mathrm{~K}$. Obviously, the saturation pressures computed with both methods converge to the same value. The moving 

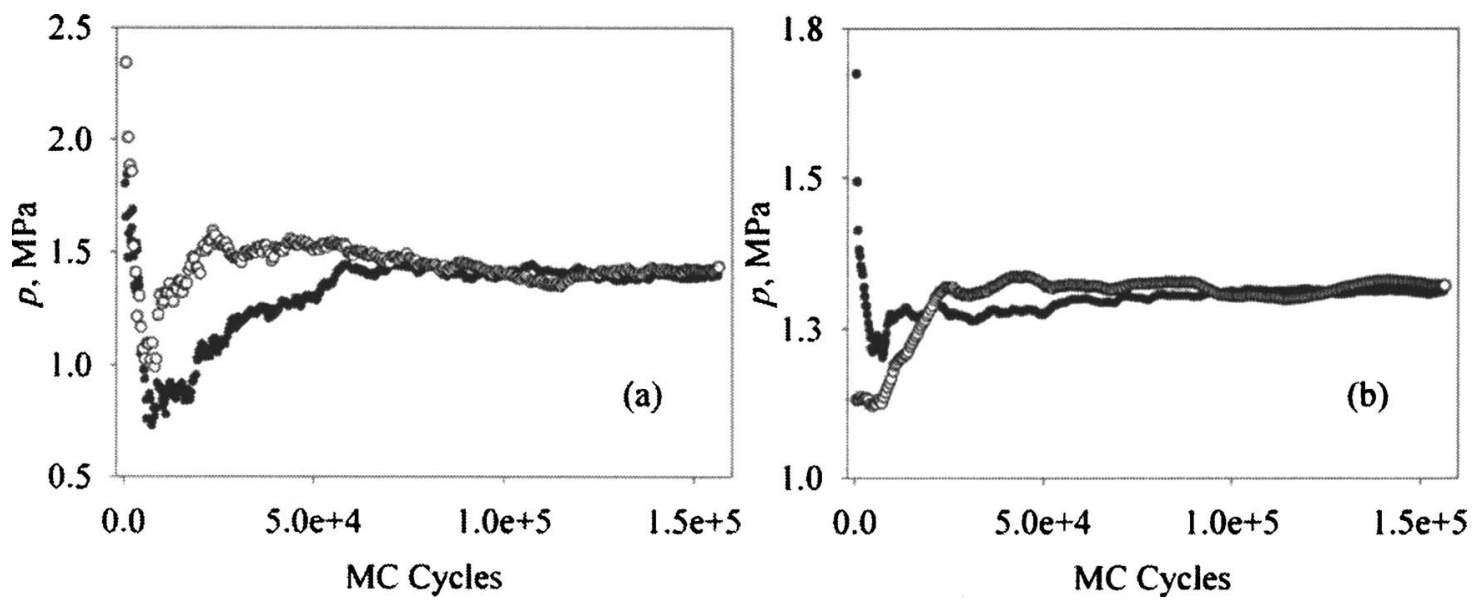

FIG. 1. Moving average of the saturation pressure of pure ethane, obtained from a $N V \beta$ Gibbs ensemble simulation at $T=250 \mathrm{~K}$. (a) Liquid-phase saturation pressure. The symbols $\bigcirc$ and $\bullet$, respectively, represent simulation results obtained from volume perturbations and from the virial. (b) Same as (a) but now for the vapor phase.

average obtained from volume perturbations converges much faster, both expressed in CPU demands and in MC cycles.

The $N p \beta+$ test molecule method with overlapping distributions provides the pure-component saturation pressure at the temperature of interest from a series of simulations. Although it is also possible to predict the liquid- and vaporphase densities at the predicted saturation pressure from a Taylor-series expansion, ${ }^{67}$ in this work we compute them from a new $N p \beta$ simulation. Anyway, a new simulation is needed to compute the initial slope of the Clapeyron equation.

Simulation results, obtained with the $N p \beta+$ test molecule method with overlapping distributions, for DMSO at $T$ $=303.15 \mathrm{~K}$ are presented in graphical form in Fig. 2. The distributions $f_{\text {per }}$ and $f_{\text {ref }}$ [see Eq. (13)] have a region of overlap from which the liquid-phase reference configurational chemical potential $\mu_{0}^{\prime L}$ can be computed. The computed Taylor-series expansions of the liquid- and vaporphase configurational chemical potentials around their reference pressures are presented in Fig. 2(b). The statistical error of the computed saturation pressure is governed by the statistical error of the liquid-phase configurational chemical potential at its reference point. The statistical errors of the results are relatively high. Better results may be obtained in case longer simulations are performed.

Computation of the configurational chemical potential from simple Widom test-molecule insertions is hardly possible at the temperatures of binary systems 3-6 in Table I. The prediction of one coexistence point with the $N p \beta+$ test molecule method with overlapping distributions is a timeconsuming task as it requires four parallel simulations per iteration. Furthermore, the computation of the Rosenbluth factor is computationally very demanding. Nevertheless, the combination of the $N p \beta+$ test molecule method with overlapping distributions and Rosenbluth sampling is very well capable of predicting vapor-liquid coexistence points at conditions where the $N V \beta$ Gibbs ensemble and the conventional $N p \beta+$ test molecule method fail.

\section{Initial slope}

A comparison of the Widom test-molecule method, difference method, and overlapping-distributions difference
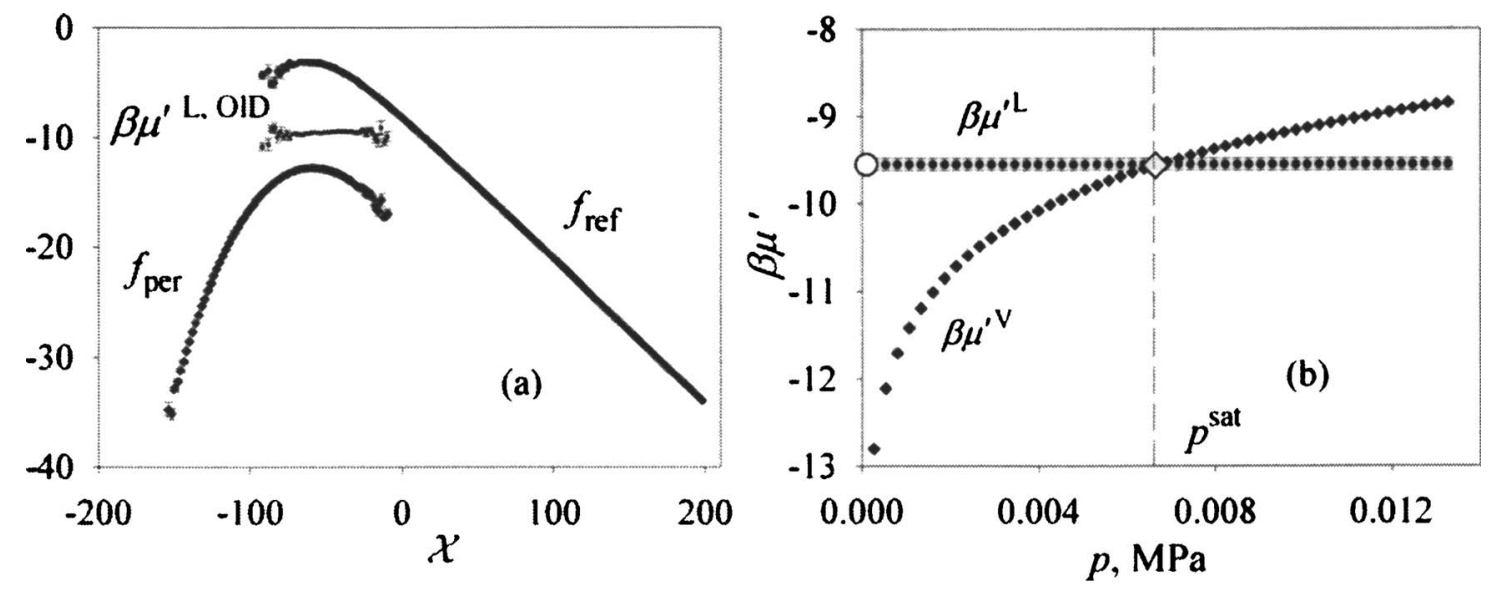

FIG. 2. Computation of a vapor-liquid coexistence point with the overlapping-distributions $N p \beta+$ test molecule method. These results have been obtained for pure DMSO at $T=303.15 \mathrm{~K}$. Error bars are given in both figures. (a) Overlapping distributions and liquid-phase configurational chemical potential. (b) Graphical representation of the liquid- and vapor-phase Taylor-series expansions of the configurational chemical potential. The symbols $\bigcirc$ and $\diamond$, respectively, represent the configurational chemical potentials at the liquid- and vapor-phase reference pressures. 
TABLE II. Liquid-phase residual chemical-potential differences at infinite dilution of a solute (2) in a solvent (1), computed with different methods at vapor-liquid coexistence conditions.

\begin{tabular}{llccc}
\hline \hline & & \multicolumn{3}{c}{$\beta \Delta \mu_{21}^{\text {res }}$ from different methods } \\
\cline { 3 - 5 } System & Condition & $\begin{array}{c}\text { Widom } \\
(-)\end{array}$ & $\begin{array}{c}\text { Difference method } \\
(-)\end{array}$ & $\begin{array}{c}\text { Overlapping distributions } \\
(-)\end{array}$ \\
\hline $\mathrm{C}_{2} \mathrm{H}_{6}(1) / \mathrm{CH}_{4}(2)$ & $250 \mathrm{~K}, 1.29 \mathrm{MPa}$ & $2.24(2)$ & $2.54(11)$ & $2.26(5)$ \\
$\mathrm{CH}_{4}(1) / \mathrm{CO}_{2}(2)$ & $150 \mathrm{~K}, 1.13 \mathrm{MPa}$ & $-1.89(2)$ & $-1.70(3)$ & $-1.90(4)$ \\
$\mathrm{C}_{3} \mathrm{H}_{8}(1) / \mathrm{CH}_{4}(2)$ & $270 \mathrm{~K}, 0.433 \mathrm{MPa}$ & $3.57(5)$ & $5.67(7)$ & $3.6(1)$ \\
$\mathrm{CHF}_{3}(1) / \mathrm{CO}_{2}(2)$ & $254 \mathrm{~K}, 1.42 \mathrm{MPa}$ & $0.72(2)$ & $1.31(8)$ & $0.69(4)$ \\
$\mathrm{CO}_{2}(1) / \mathrm{CH}_{2} \mathrm{~F}_{2}(2)$ & $244.3 \mathrm{~K}, 1.61 \mathrm{MPa}$ & $-0.95(7)$ & $-0.5(2)$ & $-0.96(6)$ \\
\hline \hline
\end{tabular}

method has already been made by $\mathrm{Liu}^{48}$ and by Liu and Govind. ${ }^{49,50}$ Their analyses were restricted to mixtures of single-site Lennard-Jones components. They only applied the difference method in the direction of increasing molecular size. It turned out that the three methods provide comparable results. The difference method had one order higher precision than the Widom test-molecule method had. For simple mixtures, the overlapping-distributions difference method had no advantage over the simple difference method. However, the overlapping-distributions difference method provided accurate results at higher densities than the other methods did. In this publication, the analyses performed by $\mathrm{Liu}^{48}$ and by Liu and Govind ${ }^{49,50}$ are extended to chain molecules. Subsequently, the failure of the difference method when swapping a large molecule with a smaller one is explained.

Numerical values of the liquid-phase residual chemicalpotential differences $\Delta \mu_{21}^{\text {res }}$ and the accompanying ratios $f_{1} / H_{2}$ for a couple of binary systems at vapor-liquid coexistence conditions are given in Tables II and III. The purecomponent coexistence conditions were computed from volume perturbations in the $N V \beta$ Gibbs ensemble.

An advantage of the Widom test-molecule method is its simplicity. Its application needs only minor modifications to the computer code of the ensemble of interest. However, the simplicity of the method is canceled out by the convergence characteristics in systems with moderate to high densities and/or in systems with large chain molecules. At high density, the larger part of the test-molecule insertions results in overlap of molecules with a corresponding negligible contribution to the ensemble average of the chemical potential.

Widom test-molecule insertions result in an energy distribution similar to the one obtained from a simulation in the reference system of the overlapping-distributions method [see Eq. (13), $\left.f_{\text {ref }}\right]$. The chemical potential computed from the Widom test-molecule method has large contributions from the poorly sampled low-energy tail of the energy distribution. Since the low-energy configurations are rarely encountered in high-density systems, the moving average of the chemical potential shows discontinuous behavior, and the higher the density, the more pronounced the discontinuities will be. A second drawback of the Widom approach is the separate computation of the ensemble averages of both residual chemical potentials; they both have an independent statistical uncertainty. Furthermore, several simulation studies in infinite periodic systems ${ }^{19,68-70}$ indicated that the chemical potential of single-site Lennard-Jones molecules is rather system-size dependent. Thus, one should always check whether the simulated system is large enough in order to avoid system-size dependence or one should apply a finitesize correction to the chemical potential. ${ }^{19,71}$

Special care is needed when Rosenbluth sampling is implemented in the computation of the residual chemical potential. ${ }^{32,35,72}$ The results of Rosenbluth sampling are only identical to those of nonbiased Boltzmann sampling in the limit of an infinitely long simulation. ${ }^{32}$ Instead of generating a molecular configuration with a probability proportional to its Boltzmann weight, the Rosenbluth scheme accepts a configuration based on its Rosenbluth weight. Especially for large chain molecules, the elongated configurations are sampled poorly. However, as this work involves only small chain molecules, this limitation is less relevant.

Liquid-phase densities at the physical conditions presented in Table II are moderate. Accordingly, the Widom test-molecule method can be efficiently used to compute the residual chemical-potential difference with high precision within reasonable time. The simulation results for the Widom

TABLE III. Liquid-phase ratios of solvent's (1) fugacity over solute's (2) Henry's constant computed with different methods at vapor-liquid coexistence conditions.

\begin{tabular}{lllcc}
\hline \hline & & \multicolumn{3}{c}{$f_{1} / H_{2}$ from different methods } \\
\cline { 3 - 5 } System & Condition & \multicolumn{1}{c}{$\begin{array}{c}\text { Widom } \\
(-)\end{array}$} & $\begin{array}{c}\text { Difference method } \\
(-)\end{array}$ & $\begin{array}{c}\text { Overlapping distributions } \\
(-)\end{array}$ \\
\hline $\mathrm{C}_{2} \mathrm{H}_{6}(1) / \mathrm{CH}_{4}(2)$ & $250 \mathrm{~K}, 1.29 \mathrm{MPa}$ & $0.107(2)$ & $0.08(10)$ & $0.105(6)$ \\
$\mathrm{CH}_{4}(1) / \mathrm{CO}_{2}(2)$ & $150 \mathrm{~K}, 1.13 \mathrm{MPa}$ & $6.6(1)$ & $5.4(1)$ & $6.7(2)$ \\
$\mathrm{C}_{3} \mathrm{H}_{8}(1) / \mathrm{CH}_{4}(2)$ & $270 \mathrm{~K}, 0.433 \mathrm{MPa}$ & $0.028(1)$ & $0.0034(2)$ & $0.028(3)$ \\
$\mathrm{CHF}_{3}(1) / \mathrm{CO}_{2}(2)$ & $254 \mathrm{~K}, 1.42 \mathrm{MPa}$ & $0.48(1)$ & $0.27(2)$ & $0.50(2)$ \\
$\mathrm{CO}_{2}(1) / \mathrm{CH}_{2} \mathrm{~F}_{2}(2)$ & $244.3 \mathrm{~K}, 1.61 \mathrm{MPa}$ & $2.6(2)$ & $1.6(3)$ & $2.6(2)$ \\
\hline \hline
\end{tabular}




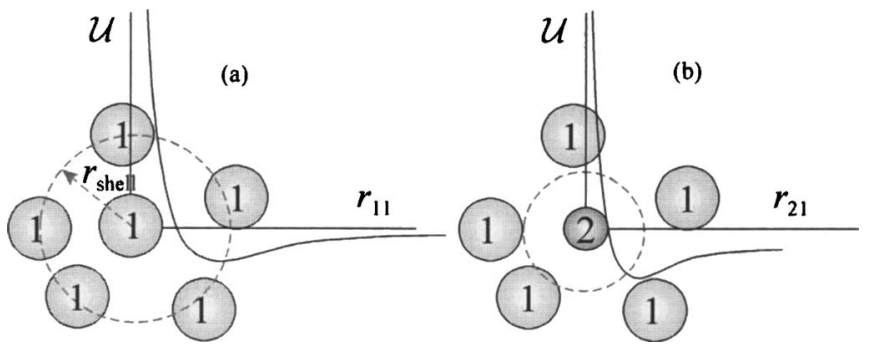

FIG. 3. Molecular configurations. The solid curve represents the intermolecular potential. The symbols $\mathcal{U}, r_{i j}$, and $r_{\text {shell }}$, respectively, represent the intermolecular energy, the intermolecular distance, and the most favorable intermolecular distance. (a) Favorable configuration of a reference system consisting of molecules with identity 1. (b) Favorable configuration of a perturbed system in which the central molecule is replaced by a molecule with identity 2 .

test-molecule method agree within their statistical errors with the results obtained from the overlapping-distributions difference method. However, the results do not match those obtained with the difference method.

The main advantage of the difference method is its easy application in a simulation; the computer code needs hardly to be changed. Since the virtual swap between an existing molecule and a molecule of another component takes place at the same center-of-mass coordinates, there is no serious insertion problem like in the Widom test-molecule method. Therefore, the method is applicable at higher densities than the simple Widom approach is. However, this advantage is less apparent when Rosenbluth sampling is used in the computation of the residual chemical potential.

The difference method determines $\Delta \mu_{21}^{\text {res,Diff }}$ as a single ensemble average. For mixtures consisting of similar components, the statistical uncertainty of $\Delta \mu_{21}^{\text {res,Diff }}$ can be much smaller than the sum of the statistical uncertainties of the individual values of $\mu_{1}^{\text {res, Widom }}$ and $\mu_{2}^{\text {res, Widom }} \cdot{ }^{43,47,54}$ Furthermore, the residual chemical-potential difference computed with the difference method seems to be less size dependent than its counterpart obtained from the Widom test-molecule method. ${ }^{47,55}$

The difference method does not provide the correct value of the residual chemical-potential difference in case a large molecule is swapped with a smaller one. ${ }^{55,73}$ However, the method works quite well the other way around in systems comprising single-site Lennard-Jones molecules. This asymmetry of perturbation methods is well known. For example, when computing the chemical potential by perturbing the ensemble of interest, insertion of test molecules usually provides the right value, while removal of real molecules does not. To explain the asymmetry of the perturbation in the difference method, we consider a perturbed system (subscript "per") and an unperturbed reference system (subscript "ref"). The reference system contains only molecules of component 1 , while the perturbed system has a molecule of component 1 replaced by a molecule of component 2 . The reference system and the perturbed system are schematically shown in Fig. 3. Suppose molecules of component 2 are smaller than those of component 1.

Favorable configurations in the reference system have configurations that look like the one shown in Fig. 3(a); the first "shell" of molecules is on average a distance $\left\langle r_{11}\right\rangle$ $\approx r_{\text {shell }}$ apart from the central molecule. This distance roughly corresponds to the distance at which the interaction energy between molecules of type 1 reaches its minimum value. Configurations with $\left\langle r_{11}\right\rangle \ll r_{\text {shell }}$ are highly improbable as they result in overlap of molecules.

The central molecule in the perturbed system is replaced by a molecule of type 2 . The interaction energy between a molecule of type 2 and a molecule of type 1 has a minimum at a distance $\left\langle r_{21}\right\rangle<r_{\text {shell }}$. This is a fair approximation when a molecule of type 2 is smaller than a molecule of type 1 . The central molecule in the perturbed system [see Fig. 3(b)] prefers the molecules of type 1 to be closer than $r_{\text {shell }}$. So, part of the configurations that is important to the perturbed system will never be sampled in the reference system. The part being never sampled has large contributions to the ensemble average. Thus, the ensemble averages of $\Delta \mu_{21}^{\text {res,Diff }}$ and $\left(f_{1} / H_{2}\right)^{\text {Diff }}$ will, respectively, be systematically higher and lower than their true values in case a large molecule is swapped with a smaller one.

The discussion of the difference method in previous publications has been restricted to mixtures of single-site Lennard-Jones molecules, ${ }^{43,49,50,55}$ or to rigid Lennard-Jones dumbbells. ${ }^{53}$ From the discussion in this section, it became
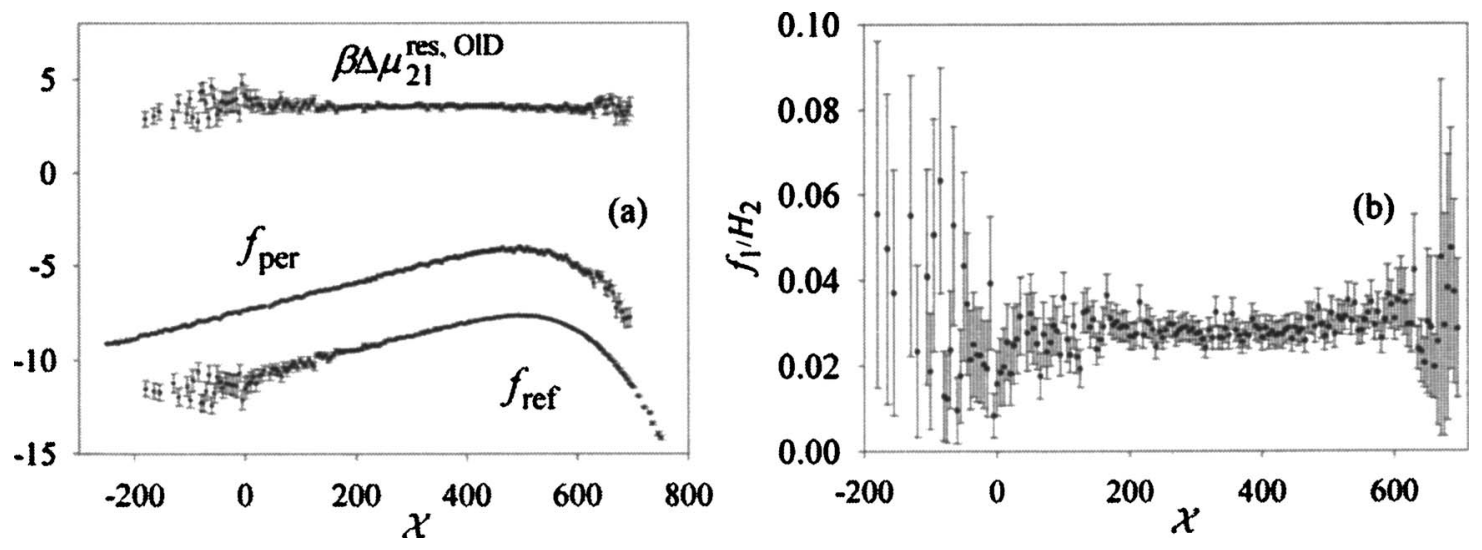

FIG. 4. Simulation results obtained with the overlapping-distributions difference method for the liquid phase of the binary system propane(1)/methane(2) at $T=270 \mathrm{~K}$ and $p=0.4333 \mathrm{MPa}$. Error bars are given in both figures. (a) Residual chemical-potential difference and overlapping distributions. (b) $f_{1} / H_{2}$ computed from $\beta \Delta \mu_{21}^{\text {res, OID }}$. 

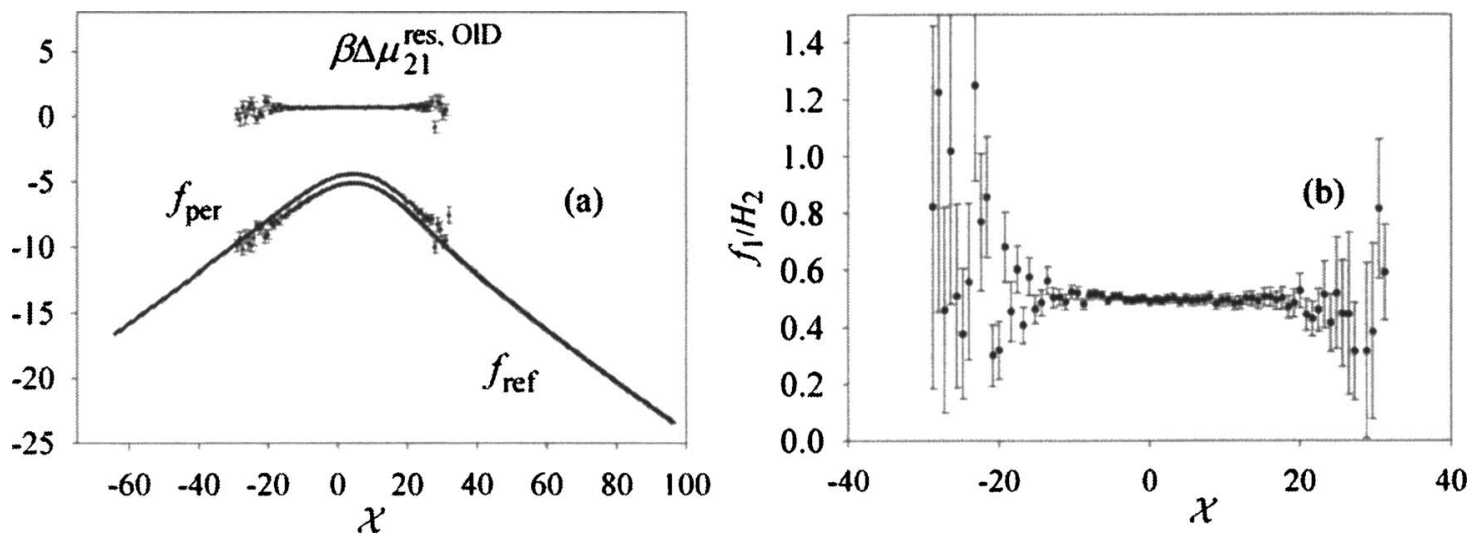

FIG. 5. Simulation results obtained with the overlapping-distributions difference method for the liquid phase of the binary system trifluoromethane(1)/carbon dioxide(2) at $T=254 \mathrm{~K}$ and $p=1.42 \mathrm{MPa}$. Error bars are given in both figures. (a) Residual chemical-potential difference and overlapping distributions. (b) $f_{1} / H_{2}$ computed from $\beta \Delta \mu_{21}^{\text {res,olD }}$.

clear that the unilateral difference method should not be used when a large single-site molecule is swapped with a smaller one. Notice that larger or smaller can hardly be defined for molecules with nonspherical geometry and intramolecular flexibility, as distances between interaction sites are orientation dependent in chain molecules.

Now, consider the simulation results presented in Tables II and III. Propane and methane are both modeled with the all-atom TraPPE-EH force field. ${ }^{58}$ Propane is a larger molecule than methane, although this difference is less clear than it is for single-site molecules. From previous arguments, it is known that the difference method does not provide the correct value of the residual chemical-potential difference in case a propane molecule is swapped with a methane molecule. Indeed, as is clear from Tables II and III, $\Delta \mu_{21}^{\text {res,Diff }}$ is much larger than $\Delta \mu_{21}^{\text {res, Widom }}$ and $\Delta \mu_{21}^{\text {res,OID }}$.

For the other couples of molecules in Table II, it is not apparent which one is the larger or the smaller one. Moreover, the molecules mentioned in Table II have rather different force-field parameters. All simulation results for $\Delta \mu_{21}^{\text {res,Diff }}$ are significantly larger than those obtained from the other methods. So, it is better not to use the difference method at all.

The overlapping-distributions difference method is applicable at higher densities than conventional unilateral perturbation methods such as the simple difference method or the Widom test-molecule method. In case the region of overlap of the distributions is large enough, the residual chemical-potential difference can be computed from those parts of the distributions that are frequently sampled by both the perturbed and the reference system, resulting in a more accurate estimate. As the overlapping-distributions difference method is a bilateral perturbation method, two simulations are needed. Implementation in the simulation code is somewhat more complicated than it is for the Widom testmolecule method or for the difference method.

Simulation results for the binary system propane/ methane at $T=270 \mathrm{~K}$ are shown in graphical form in Fig. 4 . Simulation results for the binary system trifluoromethane/ carbon dioxide at $T=254 \mathrm{~K}$ are presented in Fig. 5. The residual chemical-potential differences from the overlappingdistributions difference method, along with the statistical errors, have been determined by averaging the values in the region of overlap of the distributions. From Figs. 4(a) and $5(\mathrm{a})$, it is clear that there is a considerable overlap between the distributions $f_{\text {per }}$ and $f_{\text {ref }}$. This means that the perturbed and unperturbed systems sample to a large extent molecular configurations that are representative for each other. This enables one to compute a reliable value for the residual chemical-potential difference. The ratios $\left(f_{1} / H_{2}\right)^{\mathrm{OID}}$, along with the statistical errors, as a function of the perturbation energy $\chi$ are given in Figs. 4(b) and 5(b).

\section{Energy-biased identity changes in the semigrand- canonical ensemble}

Logically, one would think that $\gamma=1$ and $\omega=-0.5$ are suitable choices for the bias strengths, as these values remove the strongly fluctuating most-right-hand exponent in Eq. (33). However, the optimal parameters depend on the system under study. The different contributions to the bias will be considered separately in what follows.

In case the fugacity fraction of component 2 is small, the corresponding ensemble average of the mole fraction of component 2 will probably be small as well. This means that identity change $1 \rightarrow 2$ is performed much more often than the reverse change. In such a case, it may be worthwhile to choose the molecules with identity 2 more often in order to increase the overall fraction of accepted identity changes. As is clear from Eq. (33), to reach this goal, $\omega$ should be assigned a negative value.

TABLE IV. Energy-biased identity changes. Simulation results for the binary system ethane(1)/methane(2) at $T=250 \mathrm{~K}, p=1.29 \mathrm{MPa}$, and $\xi_{2}=0.10$. Percentage of accepted trial identity changes.

\begin{tabular}{lcccc}
\hline \hline Bias strength & $\begin{array}{c}1 \rightarrow 2 \\
(\%)\end{array}$ & $\begin{array}{c}2 \rightarrow 1 \\
(\%)\end{array}$ & $\begin{array}{c}\text { Overall acc. } \\
(\%)\end{array}$ & $\begin{array}{c}\pi_{1 \leftrightarrow 2} \\
(-)\end{array}$ \\
\hline$\omega=0, \gamma=0$ & 0.64 & 44 & 1.3 & $6.3 E-3$ \\
$\omega=-0.5, \gamma=0$ & 3.8 & 34 & 6.8 & $1.0 E-2$ \\
$\omega=-1, \gamma=0$ & 18 & 22 & 20 & $1.1 E-1$ \\
$\omega=-0.5, \gamma=0.25$ & 9.7 & 35 & 15 & $2.8 E-2$ \\
$\omega=-0.5, \gamma=0.5$ & 20 & 37 & 26 & $7.5 E-2$ \\
$\omega=-0.5, \gamma=1$ & 43 & 45 & 44 & $2.5 E-1$ \\
\hline \hline
\end{tabular}


TABLE V. Energy-biased identity changes. Simulation results for the binary system carbon dioxide(1)/difluoromethane(2) at $T=244.3 \mathrm{~K}, p=1.61 \mathrm{MPa}$ and $\xi_{2}=0.05$. Percentage of accepted trial identity changes.

\begin{tabular}{lcccc}
\hline \hline & $\begin{array}{c}1 \rightarrow 2 \\
(\%)\end{array}$ & $\begin{array}{c}2 \rightarrow 1 \\
(\%)\end{array}$ & $\begin{array}{c}\text { Overall acc. } \\
(\%)\end{array}$ & $\begin{array}{c}\pi_{1 \leftrightarrow 2} \\
(-)\end{array}$ \\
\hline$\omega=0, \gamma=0$ & 1.3 & 6.2 & 2.1 & $6.1 E-3$ \\
$\omega=-0.1, \gamma=0$ & 1.8 & 4.6 & 2.6 & $6.3 E-3$ \\
$\omega=-0.2, \gamma=0$ & 2.3 & 3.3 & 2.7 & $7.7 E-3$ \\
$\omega=-0.3, \gamma=0$ & 2.9 & 2.4 & 2.7 & $1.1 E-2$ \\
$\omega=-0.5, \gamma=0$ & 4.6 & 1.2 & 2.0 & $2.2 E-2$ \\
$\omega=-1, \gamma=0$ & 9.0 & 0.12 & 0.20 & $4.5 E-2$ \\
$\omega=-0.5, \gamma=0.25$ & 6.0 & 4.0 & 4.6 & $2.2 E-2$ \\
$\omega=-0.5, \gamma=0.5$ & 10 & 11 & 10 & $4.4 E-2$ \\
$\omega=-0.5, \gamma=1$ & 11 & 41 & 20 & $1.7 E-1$ \\
\hline \hline
\end{tabular}

The fugacity-fraction contribution to the bias increases the probability to select a molecule with identity 2 for $\xi_{2}$ $<0.5$ and decreases the same probability for $\xi_{2}>0.5$. However, the differences between the imposed fugacity fraction and the ensemble average of the mole fraction of component 2 can be quite large. In case $\xi_{2}>0.5$ and $\left\langle x_{2}\right\rangle \ll 0.5$, a positive value of $\omega$ is needed in order to select the molecules with identity 2 more often. Thus, the sign of $\omega$ should be selected very carefully.

The energy contribution to the bias increases the probability of changing the identity of a settled molecule with a relatively high energy. There are two things about this contribution to the bias that should be noted. At first, the ener-

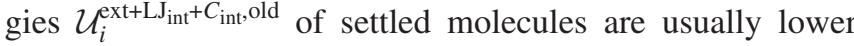
than those of new molecules that did not find a favorable orientation/position. Selection criterion (32) should not choose the molecule whose identity changed in the immediately preceding MC move too frequently. Secondly, the average energies of settled molecules with identity 1 are different from those of settled molecules with identity 2. Selection probability (32) should not choose only molecules of one component. These phenomena are a threat to ergodicity but can be weakened by choosing a moderate bias strength.

The overall fractions of accepted identity changes, obtained from isobaric semigrand-canonical simulations in the liquid phase of two different binary systems comprising chain molecules, are presented in Tables IV and V for different bias strengths. The probability $\pi_{1 \leftrightarrow 2}$ to select a molecule, given its identity changed in the immediately preceding $\mathrm{MC}$ move, is also given in Tables IV and V. Reasonableness of bias strength is assessed based on the overall fraction of accepted identity changes as well as on the probability $\pi_{1 \leftrightarrow 2}$.

In case the Coulombic energy of the molecules is computed with the Ewald summation method, ${ }^{25}$ it makes sense to exclude the reciprocal-space contribution from selection probability (32). When this is not performed, the computationally expensive $\mathbf{k}$-space contributions per molecule need to be computed during every MC move. The fractions of accepted identity changes in Table $\mathrm{V}$ are obtained by excluding the k-space contribution to the Ewald sum from the selection criterion.

Obviously, the energy bias considerably increases the fraction of accepted identity changes in the liquid phase.
However, the probability $\pi_{1 \leftrightarrow 2}$ increases as well with increasing bias strength. From Table V, it is seen that for approximately $\omega<-0.3$, the fugacity-fraction contribution to the bias has an adverse effect on the fraction of accepted identity changes. As the effect of the fugacity-fraction contribution to the bias is rather unpredictable, it is better to set $\omega$ to zero. A reasonable value for the bias strength of the energy contribution is $\gamma=0.5$. However, values of the bias strengths need to be optimized for every individual system.

\section{CONCLUSIONS}

Gibbs-Duhem integration implies the numerical integration of a Clapeyron equation. To start the numerical integration, an initial coexistence point and a corresponding initial slope of the Clapeyron equation are needed. In order to apply Gibbs-Duhem integration to all kinds of systems at diverse physical conditions, one has to investigate and assess available methods to compute these initial conditions. This publication focuses on vapor-liquid equilibria in binary mixtures comprising chain molecules.

The computation of the initial coexistence point and the corresponding initial slope often requires advanced simulation techniques in case the molar volumes of the equilibrium phases differ much, the system contains large chain molecules, and/or the size and force-field parameters of the molecules in the mixture differ much. The prediction of the initial coexistence point and the initial slope is discussed in detail.

The pure-component initial coexistence point was computed either with the $N V \beta$ Gibbs ensemble method or from a modified $N p \beta+$ test molecule method. The $N V \beta$ Gibbs ensemble method easily provides vapor-liquid coexistence points at moderate densities and for moderate differences between the liquid- and the vapor-phase molar volumes. The saturation pressure can be computed either from the virial or from volume perturbations. In case force-biased translations and torque-biased rotations are performed, for example, at moderate densities, it is convenient to compute the pressure from the virial. In other cases, one could use volume perturbations. $N V \beta$ Gibbs ensemble simulations were used to predict several pure-component saturation pressures. In general, the saturation pressure obtained from volume perturbations converged faster than its counterpart that was computed from the virial.

The modification of the $N p \beta+$ test molecule method that was introduced in this paper comprises a simulation scheme in which the configurational chemical potential is computed from the overlapping-distributions method and the Rosenbluth factor, which makes the method applicable at high densities. The method is insensitive to large differences between the molar volumes of the coexisting phases, as liquid- and vapor-phase simulations are performed independently. Although the $N p \beta+$ test molecule method with overlapping distributions is computationally very demanding, it is capable of predicting initial coexistence points at conditions where the $N V \beta$ Gibbs ensemble and the conventional $N p \beta+$ test molecule method fail.

The initial slope of the Clapeyron equation can be re- 
lated to the difference between the residual chemical potentials of a solvent and an infinitely diluted solute. We discussed three perturbation methods that can be used to predict the residual chemical-potential difference at infinite dilution: the Widom test-molecule method, the difference method, and the overlapping-distributions difference method. These simulation methods were applied to several infinitely diluted binary mixtures of chain molecules. The Widom test-molecule method and the overlapping-distributions difference method provided comparable results. The Widom test-molecule method combined with Rosenbluth sampling is applicable at relatively high densities. The overlapping-distributions difference method is applicable at even higher densities. Furthermore, it has built-in diagnostics; one can judge the reliability of the answer by investigating the overlap between the distributions. On the other hand, the difference method provides only correct answers when a small single-site molecule is perturbed to a larger one. For chain molecules, the difference method may work in neither perturbation direction, as the concepts of being smaller or larger are less clear. Thus, it is better not to use the difference method at all.

Gibbs-Duhem integration requires the frequent computation of the slope of the Clapeyron equation. This slope is computed with Monte Carlo molecular simulation in an isobaric semigrand-canonical ensemble. One of the variables that need to be sampled during a semigrand-canonical simulation is the composition. A new bias method was introduced to enhance the composition sampling of chain molecules in the semigrand-canonical ensemble. The increase in the overall fraction of accepted identity changes was considerable. The values for the bias strengths of the different contributions to the bias are most easily set to $\gamma=0.5$ and $\omega=0$. However, values of the bias strengths need to be optimized for every individual system.

${ }^{1}$ D. A. Kofke, J. Chem. Phys. 98, 4149 (1993).

${ }^{2}$ D. A. Kofke, Mol. Phys. 78, 1331 (1993).

${ }^{3}$ M. R. Hitchcock and C. K. Hall, J. Chem. Phys. 110, 11433 (1999).

${ }^{4}$ F. M. S. Silva Fernandes, R. P. S. Fartaria, and F. F. M. Freitas, Comput. Phys. Commun. 141, 403 (2001)

${ }^{5}$ R. Agrawal, Mol. Phys. 85, 43 (1995).

${ }^{6}$ R. Agrawal and D. A. Kofke, Mol. Phys. 85, 23 (1995).

${ }^{7}$ M. H. Lamm and C. K. Hall, Fluid Phase Equilib. 194-197, 197 (2002).

${ }^{8}$ M. H. Lamm and C. K. Hall, Fluid Phase Equilib. 182, 37 (2001).

${ }^{9}$ P. G. Bolhuis and D. A. Kofke, J. Phys.: Condens. Matter 8, 9627 (1996).

${ }^{10}$ J. M. Polson and D. Frenkel, J. Chem. Phys. 109, 318 (1998).

${ }^{11}$ J. M. Polson and D. Frenkel, J. Chem. Phys. 111, 1501 (1999).

${ }^{12}$ M. Mehta and D. A. Kofke, Chem. Eng. Sci. 49, 2633 (1994).

${ }^{13}$ R. Agrawal, M. Mehta, and D. A. Kofke, Int. J. Thermophys. 15, 1073 (1994)

${ }^{14}$ D. A. Kofke and E. D. Glandt, Mol. Phys. 64, 1105 (1988).

${ }^{15}$ S. S. Leung and R. B. Griffiths, Phys. Rev. A 8, 2670 (1973)

${ }^{16}$ A. van 't Hof, S. W. de Leeuw, C. K. Hall, and C. J. Peters, Mol. Phys. 102, 301 (2004).

${ }^{17}$ A. Z. Panagiotopoulos, Mol. Phys. 61, 813 (1987).

${ }^{18}$ B. Smit, P. de Smedt, and D. Frenkel, Mol. Phys. 68, 931 (1989).

${ }^{19}$ B. Smit, Ph.D. thesis, University of Utrecht, 1990.

${ }^{20}$ A. Z. Panagiotopoulos, Mol. Simul. 9, 1 (1992).

${ }^{21}$ A. Z. Panagiotopoulos, N. Quirke, M. R. Stapleton, and D. J. Tildesley,
Mol. Phys. 63, 527 (1988).

${ }^{22}$ A. Z. Panagiotopoulos, in Supercritical Fluids, Fundamentals for Applications, NATO Advanced Studies Institute, Series: Applied Science Vol. 273, edited by E. Kiran and J. M. H. Levelt Sengers (Kluwer, Dordrecht, 1994)

${ }^{23}$ D. Möller and J. Fischer, Mol. Phys. 69, 463 (1990).

${ }^{24}$ A. Lofti, J. Vrabec, and J. Fischer, Mol. Phys. 76, 1319 (1992).

${ }^{25}$ M. P. Allen and D. J. Tildesley, Computer Simulation of Liquids (Clarendon, Oxford, 1987).

${ }^{26}$ V. I. Harismiadis, J. Vorholz, and A. Z. Panagiotopoulos, J. Chem. Phys. 105, 8469 (1996)

${ }^{27}$ J. Vrabec and J. Fischer, AIChE J. 43, 212 (1997).

${ }^{28}$ G. Kronome, I. Szalai, M. Wendland, and J. Fischer, J. Mol. Liq. 85, 237 (2000).

${ }^{29}$ D. Boda, J. Liszi, and I. Szalai, Chem. Phys. Lett. 235, 140 (1995).

${ }^{30}$ J. Vrabec, A. Lofti, and J. Fischer, Fluid Phase Equilib. 112, 173 (1995).

${ }^{31}$ J. Vrabec and J. Fischer, Mol. Phys. 85, 781 (1995).

${ }^{32}$ D. Frenkel and B. Smit, Understanding Molecular Simulation; From Algorithms to Applications, Computational Science Series Vol. 1, 2nd ed., (Academic, London, 2002).

${ }^{33}$ K. S. Shing and K. E. Gubbins, Mol. Phys. 46, 1109 (1982).

${ }^{34}$ K. S. Shing and K. E. Gubbins, Mol. Phys. 49, 1121 (1983).

${ }^{35}$ G. C. A. M. Mooij and D. Frenkel, J. Phys.: Condens. Matter 6, 3879 (1994).

${ }^{36}$ A. van 't Hof, Ph.D. thesis, TU Delft, Delft, 2005.

${ }^{37}$ P. Attard, J. Chem. Phys. 103, 9884 (1995).

${ }^{38}$ D. S. Corti, Phys. Rev. E 64, 016128 (2001).

${ }^{39}$ D. S. Corti, Mol. Phys. 100, 1887 (2002).

${ }^{40}$ D. S. Corti and G. Soto-Campos, J. Chem. Phys. 108, 7959 (1998).

${ }^{41}$ K.-K. Han and H. S. Son, J. Chem. Phys. 115, 7793 (2001).

${ }^{42}$ B. Smit, Mol. Phys. 85, 153 (1995).

${ }^{43}$ P. Sindzingre, G. Ciccotti, C. Massobrio, and D. Frenkel, Chem. Phys. Lett. 136, 35 (1987).

${ }^{44}$ B. Widom, J. Chem. Phys. 39, 2808 (1963).

${ }^{45}$ B. Widom, J. Phys. Chem. 86, 869 (1982).

${ }^{46}$ K. S. Shing, Chem. Phys. Lett. 119, 149 (1985)

${ }^{47}$ K. S. Shing, J. Chem. Phys. 85, 4633 (1986).

${ }^{48}$ A. Liu, Mol. Phys. 89, 1651 (1996).

${ }^{49}$ A. Liu and R. Govind, Mol. Phys. 83, 779 (1994).

${ }^{50}$ A. Liu and R. Govind, Mol. Simul. 15, 47 (1995).

${ }^{51}$ A. Liu and Th. L. Beck, J. Chem. Phys. 105, 2424 (1996).

${ }^{52}$ A. Liu and Th. L. Beck, J. Phys. Chem. 100, 16002 (1996).

${ }^{53}$ A. Liu, Mol. Simul. 17, 75 (1996).

${ }^{54}$ D. M. Heyes, Chem. Phys. 159, 149 (1992).

${ }^{55}$ P. Sindzingre, C. Massobrio, G. Ciccotti, and D. Frenkel, Chem. Phys. 129, 213 (1989).

${ }^{56}$ J. G. Powles, W. A. B. Evans, and N. Quirke, Mol. Phys. 46, 1347 (1982).

${ }^{57}$ C. H. Bennett, J. Comput. Phys. 22, 245 (1976).

${ }^{58}$ B. Chen and J. I. Siepmann, J. Phys. Chem. B 103, 5370 (1999).

${ }^{59}$ J. J. Potoff and J. I. Siepmann, AIChE J. 47, 1676 (2001).

${ }^{60}$ B. G. Rao and U. C. Singh, J. Am. Chem. Soc. 112, 3803 (1990).

${ }^{61}$ S. Higashi and A. Takada, Mol. Phys. 92, 641 (1997).

${ }^{62}$ W. Song, N. Patel, and M. Maroncelli, J. Phys. Chem. B 106, 8783 (2002).

${ }^{63}$ C. Pangali, M. Rao, and B. J. Berne, Chem. Phys. Lett. 55, 413 (1978).

${ }^{64}$ M. Rao, C. Pangali, and B. J. Berne, Mol. Phys. 37, 1773 (1979).

${ }^{65}$ H. Flyvbjerg and H. G. Petersen, J. Chem. Phys. 91, 461 (1989).

${ }^{66} \mathrm{~B}$. Efron, The Jackknife, the Bootstrap and Other Resampling Plans (Society for Industrial and Applied Mathematics, Philadelphia, PA, 1982).

${ }^{67}$ D. Möller and J. Fischer, Fluid Phase Equilib. 100, 35 (1994).

${ }^{68}$ D. J. Adams, Mol. Phys. 28, 1241 (1974).

${ }^{69}$ U. Heinbuch and J. Fischer, Mol. Simul. 1, 109 (1987).

${ }^{70}$ B. Guillot and Y. Guissani, Mol. Phys. 54, 455 (1985).

${ }^{71}$ J. I. Siepmann, I. R. McDonald, and D. Frenkel, J. Phys.: Condens. Matter 4, 679 (1992).

${ }^{72}$ J. Batoulis and K. Kremer, J. Phys. A 21, 127 (1988).

${ }^{73}$ A. P. Malanoski and P. A. Monson, J. Chem. Phys. 112, 2870 (2000). 